\title{
Multichannel Along-Track Interferometric SAR Systems: Moving Targets Detection and Velocity Estimation
}

\author{
Alessandra Budillon, Vito Pascazio, and Gilda Schirinzi \\ Dipartimento per le Tecnologie, Università di Napoli "Parthenope”, Centro Direzionale di Napoli, Isola C4, 80143 Napoli, Italy \\ Correspondence should be addressed to Vito Pascazio, vito.pascazio@uniparthenope.it
}

Received 19 February 2008; Revised 31 July 2008; Accepted 25 September 2008

Recommended by Simon Watts

\begin{abstract}
Along-track interferometric synthetic aperture radar (AT-InSAR) systems are used to estimate the radial velocity of targets moving on the ground, starting from the interferometric phases, obtained by the combinations of two complex SAR images acquired by two antennas spatially separated along the platform moving direction. Since the radial velocity estimation obtained from a single-phase interferogram (single-channel) suffers from ambiguities, multichannel AT-InSAR systems using more than one interferogram can be used. In this paper, we first analyze the moving target detection problem, evaluating the systems performance in terms of probability of detection and probability of false alarm obtained with different values of target radial velocity, signal-to-clutter ratio, and clutter-to-thermal noise ratio. Then, we analyze the radial velocity estimation accuracy in terms of Cramer-Rao lower bounds and of mean square error values, obtained by using a maximum likelihood estimation technique. We consider the cases of single-baseline and dual-baseline satellite systems, and we evaluate the detection and estimation performance improvement obtained in the dual-baseline case with respect to the single-baseline one. Sensitivity of the presented method with respect to the involved target and system parameters is also discussed.
\end{abstract}

Copyright (C) 2008 Alessandra Budillon et al. This is an open access article distributed under the Creative Commons Attribution License, which permits unrestricted use, distribution, and reproduction in any medium, provided the original work is properly cited.

\section{INTRODUCTION}

In this paper, we review the problem of detecting the presence of a ground moving target and estimating its radial velocity by means of along-track inteferometric synthetic aperture radar (AT-InSAR) systems, mounted on moving platforms. This kind of systems can be used, for example, for continuous (day and night and with any weather condition) traffic monitoring $[1,2]$.

The detection of moving targets on the ground by means of radar systems is addressed in literature as ground moving target indication (GMTI). GMTI is a very difficult problem due to the difficulty of separating the signal returned from a moving target from the stationary background (clutter) $[3,4]$. Several methods, based on very different approaches, have been proposed in literature. In some of them, radar detection of moving targets on the ground is accomplished by enhancing the target Doppler signature against the competing ground clutter returns. Recent clutter suppression techniques use space-time adaptive processing (STAP) [5-7], requiring more than two channels, and timefrequency processing [8], requiring high pulse repetition frequency (PRF) values. While these techniques are effective in improving the detection of fast targets, for slowly moving targets the signal from clutter separation is more critical. In particular, clutter reduction becomes more critical when the Doppler frequency shift due to the target radial velocity falls inside the clutter azimuth bandwidth. Since such bandwidth increases with the ratio between the platform velocity and the azimuth antenna dimension, which in satellite-borne case is high, the range of target velocities values which are critical to be estimated can be wide in the case of satellite systems. Moreover, spectral separation requires increased PRF values, which are not desirable for the very high data rates and PRF ambiguity problems [9].

Other systems that can be used to detect ground moving target are the AT-InSAR systems, initially introduced to study ocean currents $[10,11]$, and then used to detect slow moving objects (ships, ground vehicles) [12-15] and to estimate their radial velocity. AT-InSAR systems use more than one SAR antenna (typically two), mounted on the same platform and displaced along the platform moving direction. The information about the radial velocity of the moving target is estimated from the interferometric phase of the images. 
The accuracy obtained for the estimation of ocean currents velocity using airborne AT-InSAR sensors can be of the order of few centimeters per second [10]. These very satisfactory results can be obtained since the two images acquired with a negligible time delay are very highly correlated and all the scatterers within a resolution cell move with the same velocity. In this case, the moving target is an extended one (the sea surface), while the stationary clutter is absent. The only disturbing signal to be considered is the additive thermal noise.

Differently from the case of ocean currents estimation, when AT-InSAR techniques are applied to the detection and tracking of small targets, such as vehicles, the presence of stationary clutter has to be considered. This heavily affects the interferometric phase values and their statistical distribution, thus degrading the performance of the moving target detection and of the radial velocity estimation.

For the application of statistical techniques to the detection and estimation steps, it is necessary to compute the statistical distribution of the measured interferometric phases. In the stationary image pixels, the interferometric phase reduces to only phase noise, whose statistical distribution is well known and depends on the interferometric signals correlation (the coherence) [16]. In the image pixels where a moving target is present, the phase statistical distribution diverges from that of stationary pixels, and strongly depends on the target velocity and on the statistical model assumed for the target radar cross-section (RCS). The higher the velocity, the larger the deviation, with respect to the stationary case. Different models can be assumed for the radar response of the target. In the following we will adopt two different RCS models: a deterministic model [13], and a zero mean Gaussian model [12], underlying differences and analogies.

Another problem to be taken into account is that the interferometric phase is measured in the interval $(-\pi, \pi]$, then a phase unwrapping ( $\mathrm{PhU}$ ) operation is required to retrieve the target radial velocity. The PhU operation presents solution ambiguities when only one phase interferogram (single-channel) is used. It has already been shown in $[13,15]$ that the joint use of multichannel configurations (derived from the use of more than two interferometric images acquired with different baselines or at different working frequencies) and of classical statistical estimation techniques allows to obtain very accurate solutions and to overcome the limitations due to the presence of ambiguous solutions, intrinsic in the single-channel configurations.

In this paper, we show that AT-InSAR systems based on the use of more interferograms (multichannel) acquired with frequency or baseline diversity outperform conventional ATInSAR systems using a single interferogram. In particular, we show that even a dual-baseline system allows achieving detection and estimation performance much better than the one obtained by a single-baseline system. The results obtained in the estimation process are partially a review of what we have presented in [15]. The analysis, in terms of moving target detection and radial velocity estimation accuracy, is carried out varying the main AT-InSAR system

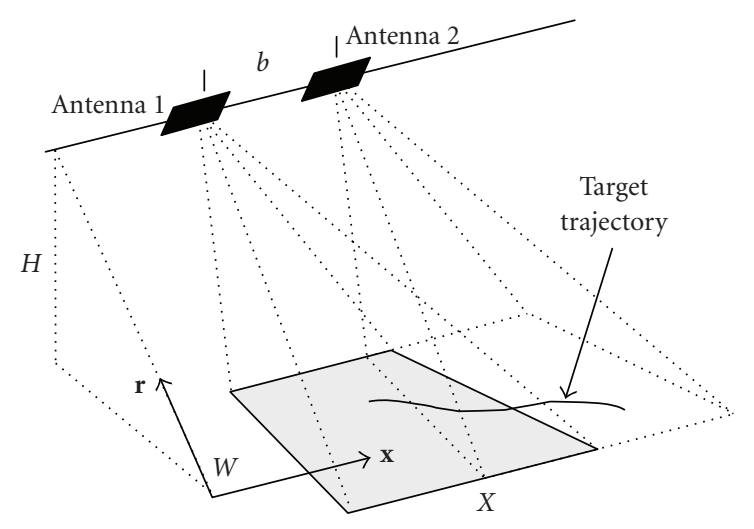

FIGURE 1: Along-track interferometry system geometry.

and target parameters, such as velocity values, signal-toclutter ratio (SCR), defined as the ratio between the power received from the moving target and the background clutter power, and clutter-to-noise ratio (CNR), defined as the ratio between the power received from the background clutter and the additive thermal noise power in the receiver. The performances presented in the following sections have been obtained considering two RCS models: deterministic and Gaussian. Finally, a discussion about robustness of the proposed model with respect to uncertainty on system parameters has been also included.

\section{ALONG-TRACK INTERFEROMETRIC SAR SYSTEMS}

Consider an AT-InSAR system constituted by two antennas moving along the direction $x$ (azimuth) (see Figure 1), and suppose that the two antennas are separated by the baseline $b$ along the azimuth direction $x$, such that $b \ll H$, where $H$ is the platform height. Assume a target on the ground moving with a constant velocity $\mathbf{v}_{T}=v_{T x} \mathbf{X}+v_{T r} \mathbf{r}$, where $v_{T x}$ and $v_{T r}$ are the velocity components along the azimuth and the line of sight direction (range) $\mathbf{r}$, respectively.

Both azimuth and range velocity components change the Doppler history of the moving target (but in different ways) in comparison to the stationary background. In order to show this behavior, we have simulated an SAR image of a stationary target shown in Figure 2(a) (the horizontal axis represents azimuth and the vertical axis represents range). In Figures 2(b) and 2(c), the images of a target moving with only radial velocity and with only azimuth velocity are shown, respectively. Finally, the image in Figure 2(d) is related to a target moving with both radial and azimuth velocity components. We can observe that the radial velocity component $v_{T r}$ produces an azimuth displacement of the target, due to a Doppler offset (see Figures 2(b) and 2(d)). The azimuth velocity component $v_{T x}$, instead, produces a Doppler slope change $[3,5]$ causing a defocusing in the moving target image (see Figures 2(c) and 2(d)) that can be compensated by using autofocusing techniques [17]. These effects can be exploited to separate moving target from stationary background by means of Doppler filtering [4]. However, Doppler filtering is effective for fast moving targets 
and requires PRF values much higher than the Doppler bandwidth for making available a certain visibility region in the frequency domain and to achieve a consistent clutter reduction.

To avoid excessive data rates and the PRF ambiguity problem [9], it is desirable to work with low PRF values. In this case, along-track interferometric systems allow the radial velocity estimation exploiting phase information.

Suppose that $\left|v_{T x}\right|,\left|v_{T r}\right| \ll\left|v_{P}\right|$, where $\mathbf{v}_{P}=v_{P} \mathbf{X}$ is the velocity of the flying platform and $H \gg X$ and $H \gg W$, where $X$ and $W$ are the antenna footprint dimensions. Let $Z_{1}$ the complex SAR image be acquired by the first antenna and let $Z_{2}$ the complex SAR image of the same ground region be acquired by the second antenna (we understand the dependence on the pixel coordinates).

The two SAR complex images can be modeled as follows:

$$
\begin{aligned}
& Z_{1}= \begin{cases}Z_{c 1}+N_{1}+Z_{T 1} & \text { in presence of moving target, } \\
Z_{c 1}+N_{1} & \text { in absence of moving target, }\end{cases} \\
& Z_{2}= \begin{cases}Z_{c 2}+N_{2}+Z_{T 2} & \text { in presence of moving target, } \\
Z_{c 2}+N_{2} & \text { in absence of moving target, }\end{cases}
\end{aligned}
$$

where $Z_{c 1}$ and $Z_{c 2}$ are the clutter signals acquired by the two antennas, $N_{1}$ and $N_{2}$ represent the thermal noise at the receivers, and $Z_{T 1}$ and $Z_{T 2}$ denote the SAR images of the moving target produced by the two interferometric antennas. The SAR target images will exhibit a phase factor related to the radial velocity [10]:

$$
\mathrm{Z}_{T 1}=A_{1}, \quad \mathrm{Z}_{T 2}=A_{2} e^{-j \phi_{v}},
$$

where $A_{1}$ and $A_{2}$ are the target complex images and $\phi_{v}$ is the nominal ATI phase which in the above-mentioned assumptions is given by

$$
\phi_{v}=\left\langle\frac{4 \pi b}{\lambda} \frac{v_{r}}{\left|v_{P}\right|}\right\rangle_{2 \pi}=\left\langle\frac{4 \pi b}{\lambda} u_{r}\right\rangle_{2 \pi},
$$

where $\langle\cdot\rangle_{2 \pi}$ is the modulo $2 \pi$ operation, $\lambda$ is the wavelength corresponding to the working frequency $f=c / \lambda$ of the SAR system, and $u_{r}=v_{r} /\left|v_{P}\right|$ is the normalized radial velocity, where the moving target is present $v_{r} \neq 0$ and $\phi_{v} \neq 0$. From (3), it follows that there are several velocity values which produce the same nominal ATI phase. The difference between two velocity values that produce the same nominal ATI phase is $2 k u_{r, \mathrm{amb}}$, where $k$ is an integer and $u_{r, \mathrm{amb}}=$ $\pm \lambda /(4 b)$ is the ambiguity velocity value corresponding to a nominal ATI phase equal to $\pm \pi$.

The SAR interferometric phase signal $\Phi$ is

$$
\Phi=\angle Z_{1} Z_{2}^{*}
$$

where $\angle$ denotes the principal phase value and $*$ denotes the conjugate.

Note that the measured interferometric phase $\phi$ differs from the nominal ATI phase $\phi_{v}$ due to the presence of clutter and noise signals.

It is well known that the SAR clutter signals $Z_{c 1}$ and $Z_{c 2}$ can be assumed as random processes, whose real and imaginary parts are mutually uncorrelated Gaussian signals, with zero mean and same variance, since they are resulting from the superposition of the signals backscattered from many scattering centers lying in the resolution cell. $N_{1}$ and $N_{2}$ can be modeled as two additive (to the clutter) zero mean Gaussian complex processes independent of each other, and independent on the clutter.

Then, when the moving target is absent, the two processes $Z_{1}$ and $Z_{2}$ are Gaussian with zero mean and correlation coefficient $\gamma$ given by [18]

$$
\begin{aligned}
\gamma & =\frac{E\left[\left(Z_{1}-E\left[Z_{1}\right]\right)\left(Z_{2}-E\left[Z_{2}\right]\right)^{*}\right]}{\sqrt{\operatorname{var}\left[Z_{1}\right] \operatorname{var}\left[Z_{2}\right]}} \\
& =\frac{E\left[\left(Z_{c 1}+N_{1}\right)\left(Z_{c 2}+N_{2}\right)^{*}\right]}{\sqrt{E\left[\left|Z_{c 1}+N_{1}\right|^{2}\right] E\left[\left|Z_{c 2}+N_{2}\right|^{2}\right]}} \\
& =\frac{\gamma_{c}}{\left(1+\sigma_{n}^{2} / \sigma_{c}^{2}\right)}=\frac{\gamma_{c}}{(1+1 / \mathrm{CNR})},
\end{aligned}
$$

where $E\left[\right.$ ] denotes the expectation operation, $\gamma_{c}$ is the clutter coherence, representing the correlation between images $Z_{c 1}$ and $Z_{c 2}$, and $C N R$ is given by

$$
\mathrm{CNR}=\frac{\sigma_{c}^{2}}{\sigma_{n}^{2}},
$$

where $2 \sigma_{c}^{2}$ and $2 \sigma_{n}^{2}$ are the clutter and thermal noise powers (the factor 2 is due to the sum of the powers of the real and imaginary parts).

In ATI-InSAR space applications, $\gamma_{c}$ is usually assumed to be equal to one [12], since the two images are acquired from the same antenna position with a time lag lower than 1 millisecond. In the case of bistatic systems, as next generation satellite clusters, clutter coherence can, instead, assume values smaller than 1. Moreover, a parasitic cross-track baseline may introduce a height-induced interferometric phase that needs to be taken into account. Anyway, it can be partly compensated by exploiting a priori DEM knowledge.

In absence of targets, the pdf of the interferometric phase can be expressed in closed form as [16]

$$
\begin{aligned}
& f_{\Phi}\left(\phi ; \phi_{0}, \gamma\right) \\
&= \frac{1}{2 \pi} \frac{1-|\gamma|^{2}}{1-|\gamma|^{2} \cos ^{2}\left(\phi-\phi_{0}\right)} \\
& \times\left\{1+\frac{|\gamma| \cos \left(\phi-\phi_{0}\right) \cos ^{-1}\left[-|\gamma| \cos \left(\phi-\phi_{0}\right)\right]}{\left[1-|\gamma|^{2} \cos ^{2}\left(\phi-\phi_{0}\right)\right]^{1 / 2}}\right\}, \\
& \phi \in(-\pi, \pi],
\end{aligned}
$$

where $\phi_{0}$ is the phase of $\gamma$, that in this case is equal to zero, being the coherence given by (5) real-valued since the real and imaginary parts of the clutter signal are uncorrelated.

When the moving target is present, two different statistical models for $Z_{T 1}$ and $Z_{T 2}$ can be considered as follows:

(1) deterministic model: the target RCS is assumed to be deterministic;

(2) Gaussian model: the target RCS is assumed to be Gaussian distributed with zero mean. 


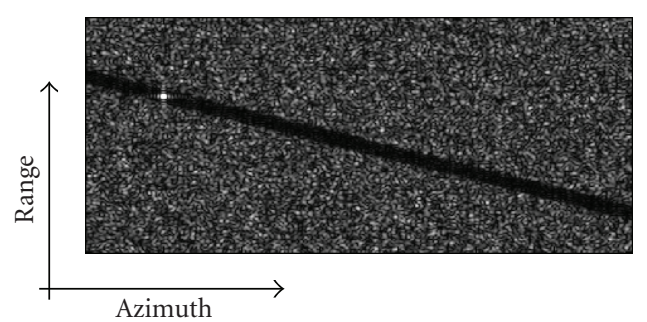

(a)

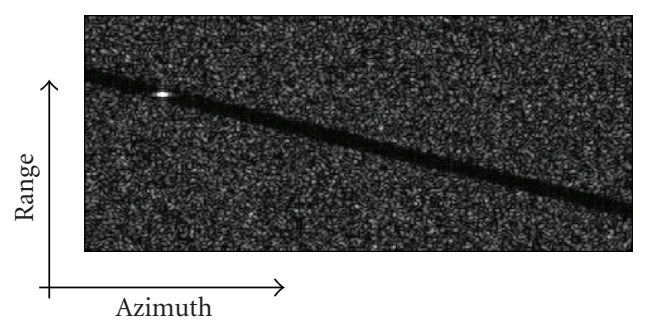

(c)

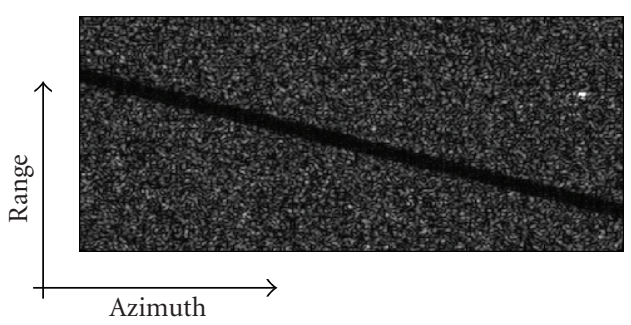

(b)

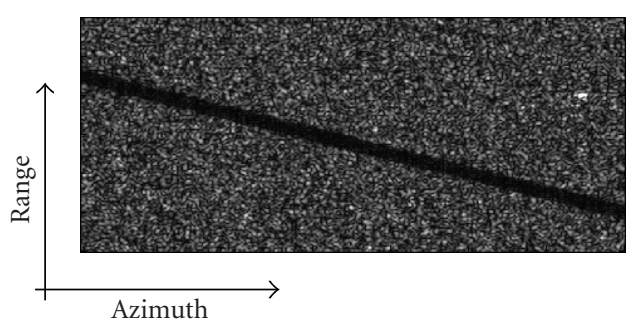

(d)

FIGURE 2: (a) Stationary target, (b) target moving with radial velocity $\left(v_{T r}=27.4 \mathrm{Km} / \mathrm{h}\right)$, (c) target moving with azimuth velocity $\left(v_{T x}=\right.$ $-109.6 \mathrm{Km} / \mathrm{h})$, and (d) target moving with both radial and azimuth velocities $\left(\mathbf{v}_{T}=v_{T x} \mathbf{x}+v_{T r} \mathbf{r}\right)$.

\subsection{Statistical distribution of AT-InSAR phase for deterministic RCS}

A deterministic model is applicable to the case of a target whose RCS can be expressed by a deterministic function of the incidenceangle. This model applies to canonical scattering objects (such as corner reflectors, spheres), and to complex or extended targets whose RCS does not rapidly change between the interferometric acquisitions. Since the RCS value of a given target mainly depends on incidence angle and target aspect angle, which does not change in the small time required to the SAR antenna to cover the baseline length, the target RCS can be usually assumed to be constant in the interferometric images. Such value influences the signal-to-clutter ratio, and is not a priori known. This is the model to be used in the interferograms simulation. It can be adopted also in the velocity estimation procedure if a precise knowledge of the RCS value is available. An accurate knowledge of the average RCS values can be available only for accurately characterized targets [19]. Moreover, this case provides the reference pdfs of the interferometric phases produced by a given complex target of known RCS. In this assumption, we can put in (2) $A_{1}=A_{2}=A$, with $A$ a deterministic constant. Then, the two processes $Z_{1}$ and $Z_{2}$ are Gaussian, with nonzero mean (due to the presence of the target) and the target RCS can be described in terms of $|A|^{2}$.

The first-order probability density function (pdf) of the interferometric phase $\Phi$ can be, in this case, numerically computed via Monte Carlo techniques, since no closed form, so far, has been found. The pdf depends on the clutter coherence coefficient $\gamma_{c}$, on the target radial velocity (as shown by (2) and (3)) and on CNR and SCR, where CNR is given by (6) and SCR is given by

$$
\operatorname{SCR}=\frac{|A|^{2}}{2 \sigma_{c}^{2}},
$$

TABLE 1: Main parameters of TerraSAR-X system.

\begin{tabular}{lc}
\hline \multicolumn{2}{c}{ TerraSAR-X } \\
\hline Height & $514.8 \mathrm{Km}$ \\
Platform velocity & $7.6 \mathrm{Km} / \mathrm{s}$ \\
Along-track antenna dimension & $4.8 \mathrm{~m}$ \\
Across-track antenna dimension & $0.8 \mathrm{~m}$ \\
Along-track baseline & $1.2 \mathrm{~m}$ \\
Working frequency & $\mathrm{X}$ band-9.65 GHz \\
Wavelength & $3.12 \mathrm{~cm}$ \\
Range bandwidth & $150 \mathrm{MHz}$ \\
\hline
\end{tabular}

where $|A|^{2}$ is the signal power. In this case, the SCR values do not affect the coherence $\gamma$ between the signals $Z_{1}$ and $Z_{2}$, which is still expressed by (5), but affect the shape of the pdf, which cannot be expressed by (7). Figure 3 shows the dependence of the pdf $f_{\Phi}(\phi)$ on SCR, CNR, and $u_{r}$ in the case of a deterministic target, evaluated for $\gamma_{c}=1$ and using the TerraSAR-X parameters [20] of Table 1. In Figure 3(a), the pdf shape is reported for the values $\mathrm{CNR}=10 \mathrm{~dB}$ and SCR $=0,10$, and $20 \mathrm{~dB}$ and a radial normalized velocity $u_{r}=3.25 \times 10^{-3}$, corresponding to the nominal ATI phase value $\phi_{v}=\pi / 2$. Figure $3(\mathrm{~b})$ is related to the values SCR $=$ $10 \mathrm{~dB}$ and $\mathrm{CNR}=0,10$, and $20 \mathrm{~dB}$ and to the same value of $u_{r}$. In Figure 3(c), the pdfs are plotted for SCR $=10 \mathrm{~dB}$ and $\mathrm{CNR}=10 \mathrm{~dB}$ and by varying the normalized velocity $u_{r}$.

Figure 3 shows that the measured phase pdfs are strongly dependent on SCR values and assume a peak value in a position which, for low SCR, is different from the nominal ATI phase value (3), that in this case is given by $\pi / 2$ and is highlighted with a dot. The dependence on CNR is, instead, less pronounced. The sensitivity of the pdfs shape with respect to $u_{r}$ is significant, and the corresponding variances gradually increase by increasing $u_{r}$. 


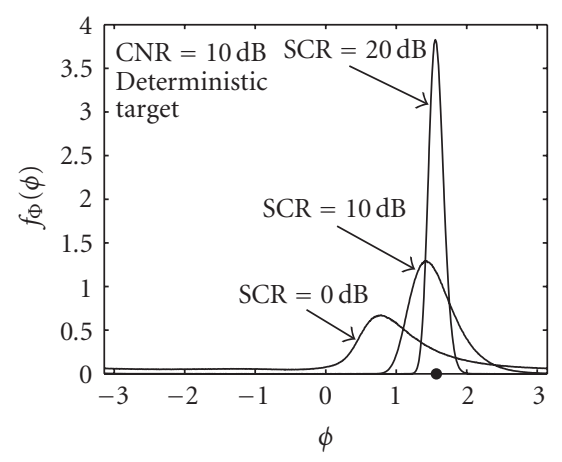

(a)

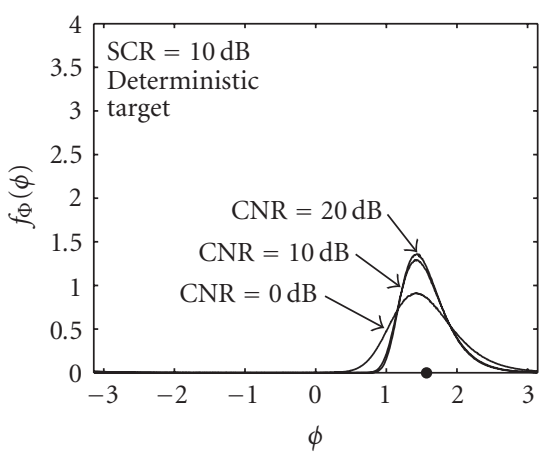

(b)

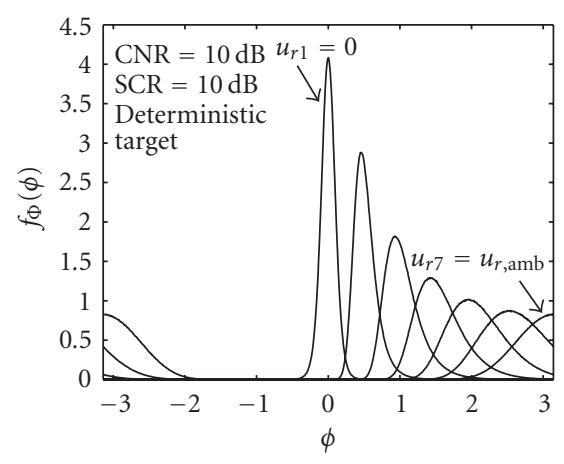

(c)

FIGURe 3: (a) Pdf of the interferometric phase in presence of a deterministic target moving with a radial velocity such that $\phi_{v}=\pi / 2($ the dot on the $\phi$ axis), for $\gamma_{c}=1, \mathrm{CNR}=10 \mathrm{~dB}$, and SCR $=0,10,20 \mathrm{~dB}$, (b) SCR $=10 \mathrm{~dB}$ and CNR $=0,10,20 \mathrm{~dB}$, and (c) SCR $=10 \mathrm{~dB}$, $\mathrm{CNR}=10 \mathrm{~dB}$, for seven radial velocities $\left(u_{r k}=(k-1) / 6 u_{r, \mathrm{amb}}, k=1, \ldots, 7\right)$.

\subsection{Statistical distribution of AT-InSAR phase for Gaussian RCS}

A Gaussian model is applicable when the RCS of the targets $A_{1}$ and $A_{2}$ and then the signals $Z_{T 1}$ and $Z_{T 2}$ are assumed to be zero mean (complex) Gaussian processes, as in [12]. This model allows to take into account the lack of knowledge of the target RCS values (that can be described in terms of variance $\sigma_{T}^{2}$ ) and then of the SCR. It applies to complex or extended targets which can be considered to consist of a large number of isotropic scattering elements, randomly distributed in a region whose dimensions are large compared to the wavelength of the illuminating radiation, and all contributing to the overall signal with the same weight [21]. When the number of the elementary scatterers in which the target can be decomposed is small and/or some of them are dominant with respect to the others, the pdf of the backscattered signal is not zero mean Gaussian and is difficult to derive [22]. However, even if this model could not be always appropriate for the description of the signal intensity distribution, it has the advantage of providing an analytical form for the interferometric phase pdf, which in many cases well approximates the true distribution. Moreover, as it will be shown in the next section, the adoption of a Gaussian model for the moving target RCS instead of the actual deterministic model will not impair significantly the GMTI performance.

Since the signals $Z_{1}$ and $Z_{2}$ acquired by the two interferometric antennas are still zero mean Gaussian signals, as happens when the target is absent, the pdf of the interferometric phase can be expressed in the closed form given by (7), where $\gamma$ is the coherence coefficient between the images $Z_{1}$ and $Z_{2}$, and $\phi_{0}$ is the phase of $\gamma$. Now, the expression of $\gamma$ is changed with respect to (5) and is given by [12]

$$
\begin{aligned}
\gamma & =\frac{E\left[\left(Z_{c 1}+N_{1}+Z_{T 1}\right)\left(Z_{c 2}+N_{2}+Z_{T 2}\right)^{*}\right]}{\sqrt{E\left[\left|Z_{c 1}+N_{1}+Z_{T 1}\right|^{2}\right] E\left[\left|Z_{c 2}+N_{2}+Z_{T 2}\right|^{2}\right]}} \\
& =\frac{\gamma_{c} \sigma_{c}^{2}+\sigma_{T}^{2} \gamma_{T}}{\sigma_{c}^{2}+\sigma_{n}^{2}+\sigma_{T}^{2}}=\frac{\gamma_{c}+\gamma_{T} \mathrm{SCR}}{1+1 / \mathrm{CNR}+\mathrm{SCR}},
\end{aligned}
$$

where SCR $=\sigma_{T}^{2} / \sigma_{c}^{2}$, and $\gamma_{T}$ is the target (complex) coherence and depends on the target velocity through the nominal phase (1):

$$
\begin{aligned}
\gamma_{T} & =\frac{E\left[Z_{T 1} Z_{T 2}^{*}\right]}{\sqrt{E\left[\left|Z_{T 1}\right|^{2}\right] E\left[\left|Z_{T 2}\right|^{2}\right]}} \\
& =\frac{E\left[A_{1} A_{2}^{*}\right]}{\sqrt{E\left[\left|A_{1}\right|^{2}\right] E\left[\left|A_{2}\right|^{2}\right]}} e^{j \phi_{v}}=\gamma_{T 0} e^{j \phi_{v}},
\end{aligned}
$$

where $\gamma_{T 0}$ is the target coherence for zero radial velocity, equal to one. It has to be noted that $\phi_{0}$, the phase of $\gamma$, is different from $\phi_{v}$.

Figure 4 shows the dependence of the pdf $f_{\Phi}(\phi)$ on SCR, $\mathrm{CNR}$, and $u_{r}$ in the case of a Gaussian distributed target, evaluated for $\gamma_{c}=\gamma_{T 0}=1$ and using the TerraSAR-X parameters [20] of Table 1. In Figure 4(a), the pdf shape is reported for the values $\mathrm{CNR}=10 \mathrm{~dB}$ and $\mathrm{SCR}=0,10$, and $20 \mathrm{~dB}$ and a radial normalized velocity $u_{r}=3.25 \times 10^{-3}$, corresponding to the nominal ATI phase value $\phi_{v}=\pi / 2$. Figure $4(\mathrm{~b})$ is related to the values $\mathrm{SCR}=10 \mathrm{~dB}$ and $\mathrm{CNR}=$ 0,10 , and $20 \mathrm{~dB}$ and to the same value of $u_{r}$. In Figure $4(\mathrm{c})$, the pdfs are plotted for SCR $=10 \mathrm{~dB}$ and $\mathrm{CNR}=10 \mathrm{~dB}$ and by varying the normalized velocity $u_{r}$.

Figure 4 shows that also in this case the measured phase pdfs are not centered on the noise-free value highlighted with a dot and given by (3), and that their shape strongly depends on SCR and weakly on CNR. Moreover, we note that now the pdfs have always a behavior that is symmetrical around the phase $\phi_{0}$, contrarily to what happened in the deterministic case (see Figure 3 ). The sensitivity of pdfs shape with respect to $u_{r}$ is similar to the deterministic case and also in this case the corresponding variances gradually increase by increasing $u_{r}$.

However, for high SCR (strongly reflective target), the pdfs derived in the two cases are quite similar, as shown in Figure 5, where we have reported the pdfs related to the two models, (a) with SCR $=0 \mathrm{~dB}$, (b) SCR $=10 \mathrm{~dB}$, and (c) $\mathrm{SCR}=20 \mathrm{~dB}$. 


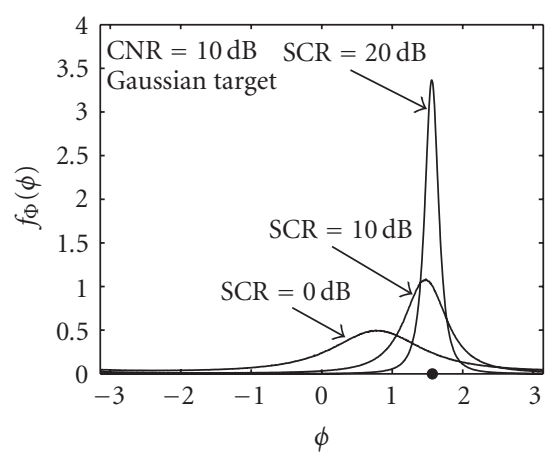

(a)

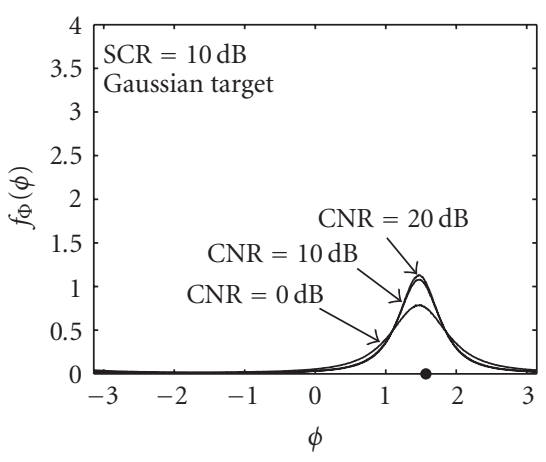

(b)

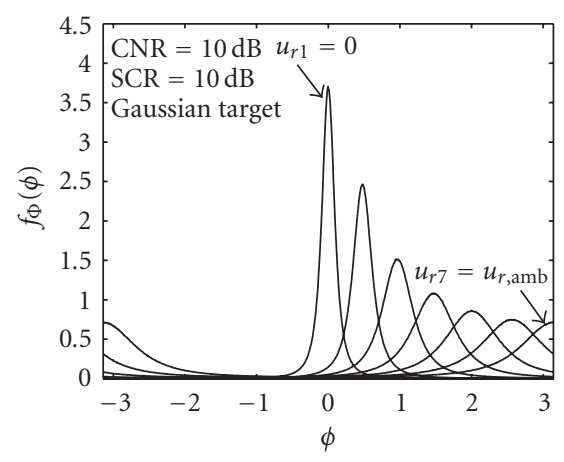

(c)

FIGURe 4: (a) Pdf of the interferometric phase in presence of Gaussian target moving with a radial velocity such that $\phi_{v}=\pi / 2($ the dot on the $\phi$ axis), for $\gamma_{c}=1, \mathrm{CNR}=10 \mathrm{~dB}$ and SCR $=0,10,20 \mathrm{~dB}$, (b) SCR $=10 \mathrm{~dB}$ and CNR $=0,10,20 \mathrm{~dB}$, and (c) SCR $=10 \mathrm{~dB}, \mathrm{CNR}=10 \mathrm{~dB}$, for seven radial velocities $\left(u_{r k}=(k-1) / 6 u_{r, \text { amb }}, k=1, \ldots, 7\right)$.

Moreover, the availability of an analytical expression for the pdf and for the coherence (see (7) and (10)) allows to easily discuss the effects that $\mathrm{CNR}, \mathrm{SCR}$, and radial velocity variations have on the interferometric measured phase distribution. First of all, we note that the pdf behavior changes according to the changes of $|\gamma|$ and $\phi_{0}$. In particular, when $|\gamma|$ decreases, the spreading of the measured phase values around $\phi_{0}$ increases, while the changes in the values of $\phi_{0}$ determine a simple circular shift of the curves. The first effect affects the velocity estimation accuracy, while the latter introduces a phase polarization $\phi_{0}-\phi_{v}$, which, if known, can be implicitly taken into account and compensated in the velocity estimation procedure.

We can easily analyze the pdf dependence on the system and target parameters in three limit cases.

\section{(i) Strong targets}

SCR $\gg 1$ and CNR $\gg 1$; then, from (9) and (10), $|\gamma| \cong\left|\gamma_{T_{0}}\right|$ is independent on the velocity value and $\phi_{0} \cong \phi_{v}$, that is, the pdf maximum position does not depend on SCR and CNR. For high SCR values we expect that the velocity estimation accuracy does not depend on the velocity value.

\section{(ii) Camouflaged targets}

SCR $\cong 1, \mathrm{CNR} \gg \mathrm{SCR}$, and assume $\gamma_{c} \cong \gamma_{T 0}$; then, from (9) and $(10), \gamma \cong \gamma_{c}\left(1+\exp \left(j \phi_{v}\right)\right) / 2=\gamma_{c} \exp \left(j \phi_{v} / 2\right) \cos \left(\phi_{v} / 2\right)$. Consequently, $|\gamma| \cong\left|\gamma_{c} \cos \left(\phi_{v} / 2\right)\right|$ and $\phi_{0} \cong \phi_{v} / 2$. In this case, the pdf spreading and the phase polarization are strongly dependent on the velocity value.

\section{(iii) Weak targets}

SCR $\ll 1$; then, from (9) and (10), $\gamma \cong \gamma_{c} /(1+$ CNR). In this case $\phi_{0} \cong 0$, the clutter term is dominant, and the pdf of the phase is in practice independent on the velocity value. As expected, the velocity estimation problem cannot be solved for very low SCR values.

The behavior of $|\gamma|$ and $\phi_{0}$ versus SCR using the TerraSAR-X parameters, with $\phi_{v}=\pi / 2$ and CNR values of 10,20 , and $30 \mathrm{~dB}$, is shown in Figure 6 . Note that $\phi_{0}$ is independent on CNR for the considered values of SCR, while $|\gamma|$ is practically independent on CNR for SCR values larger than $0 \mathrm{~dB}$. Then, we expect that for $\mathrm{SCR}$ values greater than $0 \mathrm{~dB}$, measurement errors on CNR will not influence significantly the velocity estimation accuracy.

Note further that, for small changes of SCR around the value $\mathrm{SCR}=0 \mathrm{~dB}(\mathrm{SCR}=1)$, the curves exhibit strong variations. This implies that small errors in the knowledge of the SCR can affect the velocity estimation accuracy.

For the deterministic model the pdf is not given by (6), and there is not a direct relation between $|\gamma|$ and the pdf spreading, and between $\phi_{0}$ and the position of the pdf maximum. Even so, the general qualitative behavior of $|\gamma|$ and $\phi_{0}$ with respect to changes of SCR, CNR, $\gamma_{c}$, and $\gamma_{T 0}$ is similar to the one obtained in the Gaussian case.

\section{MULTICHANNEL ALONG-TRACK SAR INTERFEROMETRY}

The along-track interferometric phase depends on radial velocity, baseline, and wavelength as shown in (3). Phase values outside interval $(-\pi, \pi)$ wrap $\bmod (2 \pi)$, so that such values are indistinguishable from the ones differing for $2 \pi$ multiples. The same holds for the corresponding radial velocity values. The radial velocity ambiguity value $u_{r, \text { amb }}=$ $\pm \lambda /(4 b)$, corresponding to the interferometric phase $\pm \pi$, is then the maximum velocity value that can be unambiguously detected. Moreover, in the realistic case of noisy data, this ambiguity problem can be present also for normalized radial velocities smaller than $u_{r, \text { amb }}$. Such effect can be particularly critical either for detection applications or for velocity estimation ones.

A method for overcoming these limitations, restoring the solution uniqueness, consists in exploiting different datasets acquired with different baselines, or with frequency diversity $[13,15]$.

Different baseline datasets (at least two) can be generated when the AT-InSAR system is constituted by more than two antennas (at least three). Different frequency datasets 


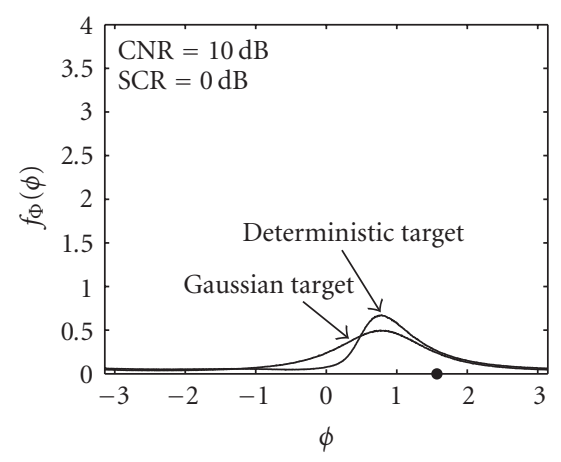

(a)

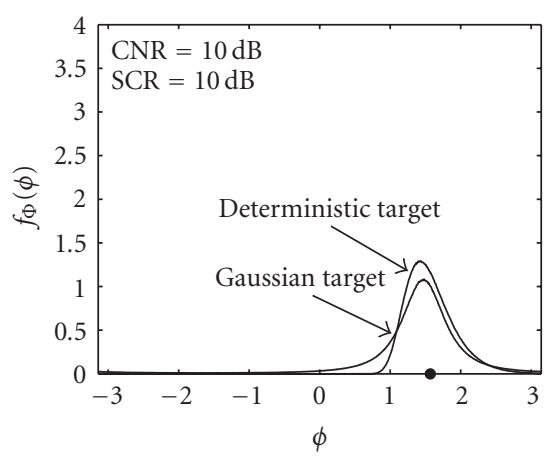

(b)

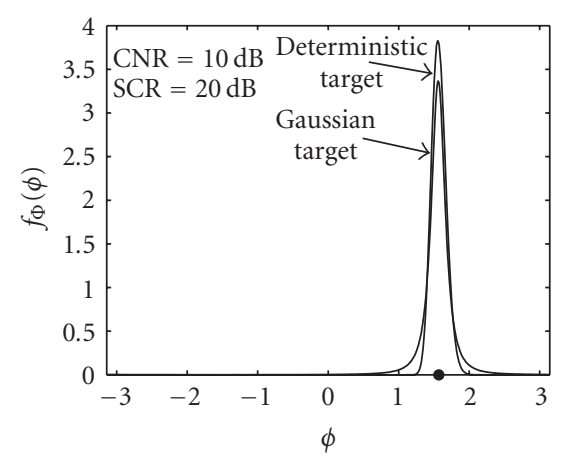

(c)

FIgURE 5: Pdfs of the interferometric phase in presence of a deterministic and a Gaussian target moving with a radial velocity such that $\phi_{v}=\pi / 2$ (the dot on the $\phi$ axis), for $\gamma_{c}=1 C N R=10 \mathrm{~dB}$ and (a) SCR $=0 \mathrm{~dB}$, (b) SCR $=10 \mathrm{~dB}$, and (c) SCR $=20 \mathrm{~dB}$.

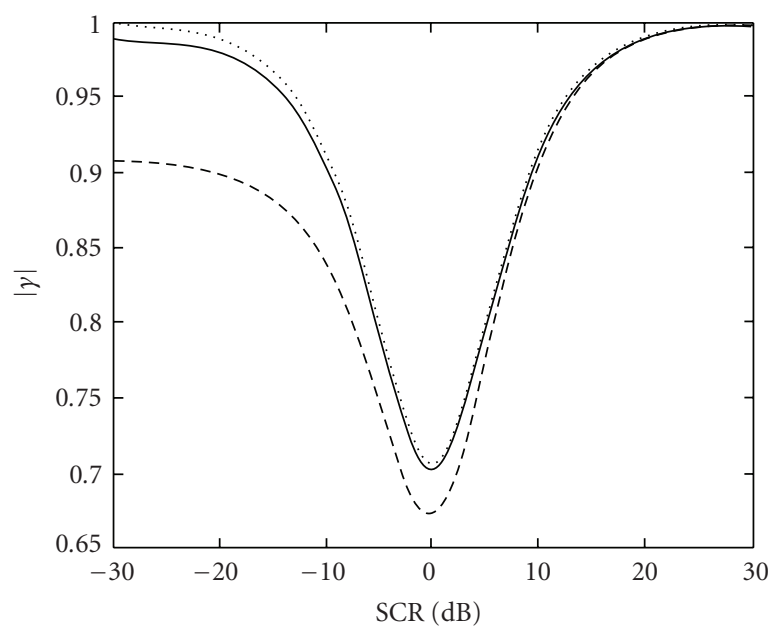

(a)

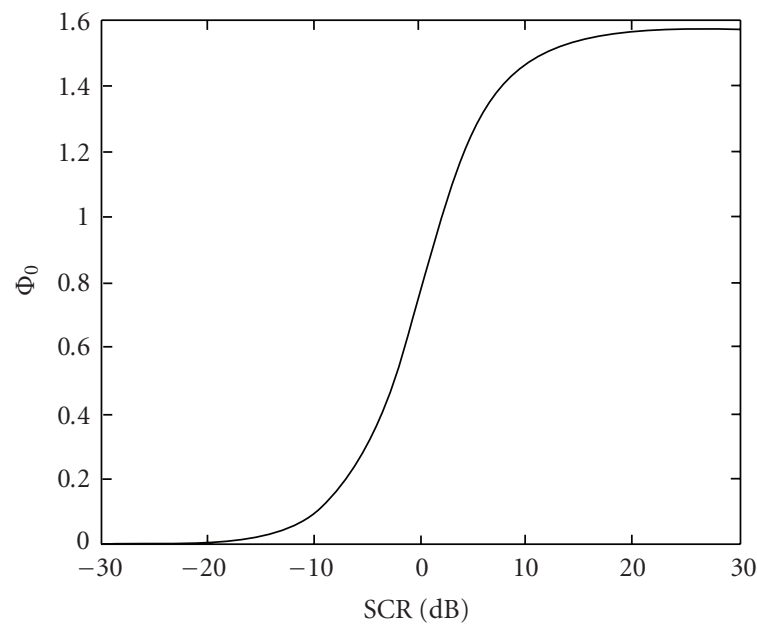

(b)

FIGURE 6: TerraSAR-X parameters and Gaussian target model: (a) $|\gamma|$ versus SCR, and (b) $\phi_{0}$ versus SCR, with CNR $=10 \mathrm{~dB}$ (dashed line), $\mathrm{CNR}=20 \mathrm{~dB}$ (solid line), $\mathrm{CNR}=30 \mathrm{~dB}$ (dotted line), and $\gamma_{c}=\gamma_{T 0}=1$. Note that the $\phi_{0}$ curves obtained for different values of CNR are coincident.

can be generated in two ways. In the first, we can suppose that the SAR sensors can operate at different working frequencies, for instance in $\mathrm{X}$ and $\mathrm{C}$ bands simultaneously. In the second, the multifrequency interferograms can be obtained by subband filtering of the interferometric images splitting the overall bandwidth as shown in Figure 7. Azimuth band partition produces the conventional azimuth looks, while range band partition produces different range looks. Note that this second partition generates looks with a small frequency diversity. Their generation is finalized to phase noise suppression as in conventional multilook procedure.

We will refer in the following to multifrequency and/or multibaseline configuration as multichannel configuration. The moving target detection and the radial velocity estimation are performed from the knowledge of such multiple wrapped interferometric phase (statistically independent) signals obtained with different baselines or with different working frequencies. It has to be noted that when the channels originate by band partition, the multichannel approach has a drawback, as the SCR is reduced, as we will show in the following. by

The CNR is given, in the case of a single-look SAR image,

$$
\begin{aligned}
\mathrm{CNR}_{\text {SLook }} & =\frac{P_{\text {Clutter }}}{P_{\text {Noise }}} \\
& =\frac{\left(K_{a} K_{r}\right)^{2}\left(P_{T x} G_{A} \lambda^{2} /(4 \pi)^{3} R^{4}\right)\left(\delta x \delta r \sigma_{0}\right)}{K_{a} K_{r} P_{\text {Noise }}},
\end{aligned}
$$

where $K_{a}$ and $K_{r}$ are the integratio samples along the azimuth and the range direction, respectively, $P_{T x}$ is the power transmitted by the SAR antenna, $G_{A}$ is the radar antenna gain, $R$ is the distance between the SAR antenna and the ground region where this ratio is evaluated, $\sigma_{0}$ is the normalized radar cross-section relative to the background (the clutter), $\delta x$ and $\delta r$ are the spatial resolutions of the images, and $P_{\text {Noise }}$ is the thermal power at the receiver. Note that the product $\left(\delta x \delta r \sigma_{0}\right)$ represents the RCS of a clutter resolution cell. 


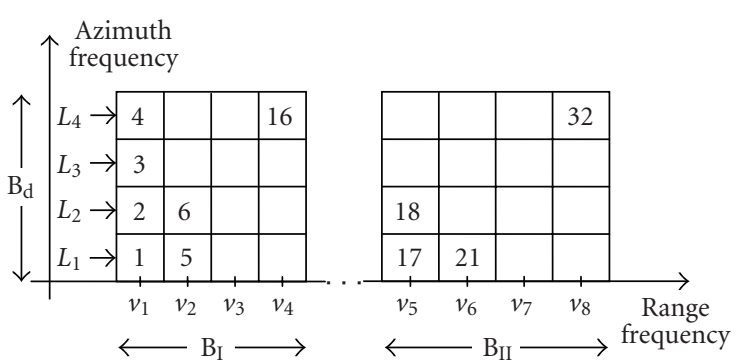

FIgURe 7: Partition of the dual-band spectrum of a hypothetical SAR interferometric system. The two bands $B_{I}$ and $B_{I I}$ are subband filtered into $N_{r}=8$ range subband (central frequencies $\left.v_{1}, v_{2}, \ldots, v_{8}\right)$, and the Doppler band $\mathrm{B}_{\mathrm{d}}$ into $N_{a}=4$ azimuth looks $\left(L_{1}, L_{2}, L_{3}, L_{4}\right)$. Each $\left(n=1, \ldots, N=N_{a} \times N_{r}=32\right)$ identifies a portion of the $2 \mathrm{D}$ frequency domain not overlapping with the others. Absence of overlapping guarantees the statistical independence of interferograms. by

The SCR is given, in the case of a single-look radar image,

$$
\begin{aligned}
\text { SCR }_{\text {SLook }} & =\frac{P_{\text {Target }}}{P_{\text {Clutter }}}=\frac{\left(K_{a} K_{r}\right)^{2}\left(P_{T x} G_{A} \lambda^{2} /(4 \pi)^{3} R^{4}\right) \sigma_{\text {Target }}}{\left(K_{a} K_{r}\right)^{2}\left(P_{T x} G_{A} \lambda^{2} /(4 \pi)^{3} R^{4}\right)\left(\delta x \delta r \sigma_{0}\right)} \\
& =\frac{\sigma_{\text {Target }}}{\left(\delta x \delta r \sigma_{0}\right)},
\end{aligned}
$$

where $\sigma_{\text {Target }}$ is the moving target RCS.

In the case of a multichannel system, two cases have to be distinguished: the case where more antennas (multibaseline) or more working frequencies are used, and the case where the overall system bandwidth is partitioned into different subbands (multilook). In the first case, the spatial resolution does not change, and supposing that the integration samples are the same for each channel, the CNR and the SCR values do not change. In the latter case, as the band partition reduces the spatial resolution, and supposing that $N_{a}$ and $N_{r}$ are the number of azimuth looks and range looks, respectively, the CNR and the SCR values change in

$$
\begin{aligned}
& \mathrm{CNR}_{\text {MLook }} \\
& \qquad=\frac{\left(K_{a} K_{r} / N_{a} N_{r}\right)^{2}\left(P_{T x} G_{A} \lambda^{2} /(4 \pi)^{3} R^{4}\right)\left(N_{a} \delta x N_{r} \delta r \sigma_{0}\right)}{\left(K_{a} K_{r} / N_{a} N_{r}\right) P_{\text {Noise }}} \\
& =\text { CNR }_{\text {SLook }},
\end{aligned}
$$

$$
\operatorname{SCR}_{\text {MLook }}=\frac{\sigma_{\text {Target }}}{\left(N_{a} \delta x N_{r} \delta r \sigma_{0}\right)}=\frac{1}{\left(N_{a} N_{r}\right)} \text { SCR }_{\text {SLook. }}
$$

The multichannel (derived by band partition) along-track SAR interferometry system scheme is depicted in Figure 8.

\section{MULTICHANNEL AT-InSAR MOVING TARGET DETECTION}

The interferometric phase $\Phi$ is distributed according to a pdf depending on several parameters:

$$
f_{\Phi}\left(\phi \mid u_{r} ; \lambda, b, \gamma_{c}, \mathrm{SCR}, \mathrm{CNR}\right) .
$$

Of course, in the absence of a moving target $\left(u_{r}=\right.$ 0 , and SCR $=0)$, $\Phi$ reduces to pure phase noise.

A moving target can be detected by comparing the interferometric phase $\Phi$ with a threshold $\phi_{T}$ in the interval $(-\pi, \pi]$. We can evaluate the detection probability $\left(P_{D}\right)$ and false alarm probability $\left(P_{\mathrm{FA}}\right)$ in the following way:

$$
\begin{aligned}
P_{D}= & \int_{-\pi}^{-\phi_{T}} f_{\Phi}\left(\phi \mid u_{r} ; \lambda, b, \gamma_{c}, \mathrm{SCR}, \mathrm{CNR}\right) d \phi \\
& +\int_{\phi_{T}}^{\pi} f_{\Phi}\left(\phi \mid u_{r} ; \lambda, b, \gamma_{c}, \mathrm{SCR}, \mathrm{CNR}\right) d \phi \\
P_{\mathrm{FA}}= & 2 \int_{\phi_{T}}^{\pi} f_{\Phi}\left(\phi \mid 0 ; \lambda, b, \gamma_{c}, \mathrm{CNR}\right) d \phi .
\end{aligned}
$$

The performance of the detection process is, as expected, better for high values of SCR, that is, when the moving targets power is significantly larger than the clutter power. For moving targets mingling with the background clutter, the detection capability worsens, so that if one wants low values of $P_{\mathrm{FA}}$, the $P_{D}$ can decrease to very low values, not consistent with the applications [23]. This approach, based on a single interferogram value, does not provide the desired results in terms of simultaneous low values of $P_{\mathrm{FA}}$ and high values of $P_{D}$. An alternative improved detection strategy is based on the use of multichannel interferograms. After the application of the threshold to each channel, a binary integration procedure can be adopted to combine singlechannel decisions.

We use a hypothetical dual-baseline system working at the frequency $f=9.65 \mathrm{GHz}$, with three antennas separated by the two baselines $b_{1}=1.2 \mathrm{~m}$ and $b_{2}=1.8 b_{1} \mathrm{~m}$. Both interferometric signals are partitioned into 4 azimuth looks, for a total of $N=8$ channels. Note that the same effect could be obtained with a dual frequency system, with a first working frequency $f_{1}$ and a second working frequency equal to $f_{2}=1.8 f_{1}$ (e.g., $\mathrm{C}$ band and $\mathrm{X}$ band).

Suppose that the detection probability of one of the channels corresponding to the first baseline (or first frequency) is equal to $P_{D 1}$, and that the detection probability of one of the channels corresponding to the second baseline (or second frequency) is equal to $P_{D 2}$; we can evaluate the probability that the target is detected from $(N / 2+j)$ channels $(j=$ $1, \ldots, N / 2)$ on a total of $N$ channels:

$$
\begin{aligned}
P_{j} & =\sum_{k=j}^{N / 2}\left[\left(\begin{array}{c}
\frac{N}{2} \\
k
\end{array}\right) P_{D 1}^{k}\left(1-P_{D 1}\right)^{N / 2-k}\right] \\
& \times\left[\left(\begin{array}{c}
\frac{N}{2} \\
\frac{N}{2}-k+j
\end{array}\right) P_{D 2}^{N / 2-k+j}\left(1-P_{D 2}\right)^{k-j}\right] .
\end{aligned}
$$

We have developed two possible strategies and compared them with the one based on a single interferogram. Strategy 1 consists in considering present the moving target when the majority of the interferogram values are above prefixed 


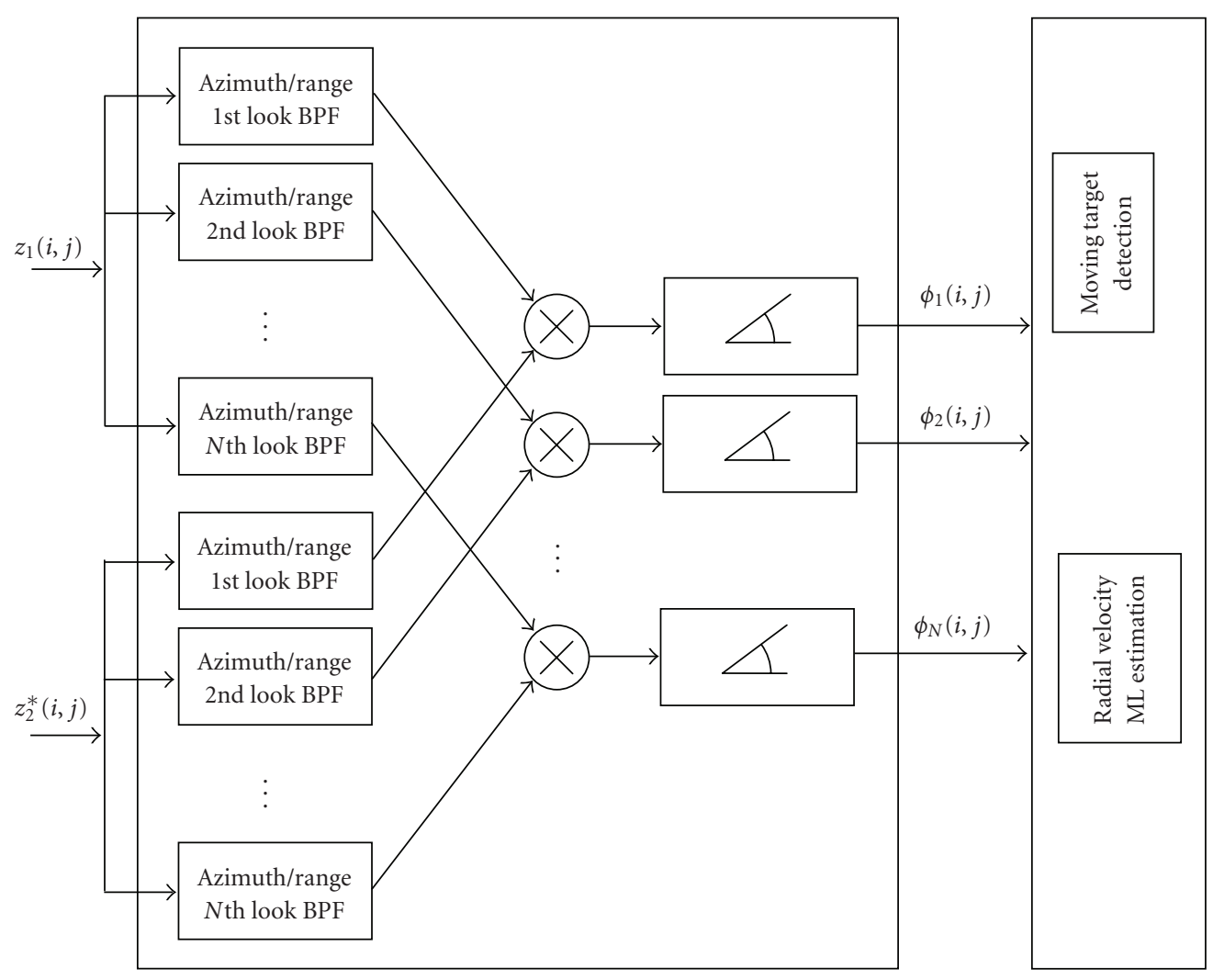

FIGURE 8: Multichannel along-track SAR interferometry system scheme.

thresholds. We obtain the following estimated detection probability:

$$
P_{D>N / 2}=\sum_{j=1}^{N / 2} P_{j} .
$$

For the estimation of the false alarm probability, we have used the same reasoning, using the single-channel false alarm probabilities $P_{\mathrm{FA} 1}$ and $P_{\mathrm{FA} 2}$ in place of $P_{D 1}$ and $P_{D 2}$ in (17).

Strategy 2 consists in considering present the moving target when more than $3 / 4$ of the total interferogram values are above prefixed thresholds. We obtain

$$
P_{D>3 N / 4}=\sum_{j=n / 4+1}^{N / 2} P_{j} .
$$

In Figure 9, we have reported all the $P_{D}$ 's corresponding to different operating conditions and in the case of the deterministic model (the Gaussian model provides similar results). We have reported also the estimation of $P_{D}$ adopting Strategy 1 (dashed line) and Strategy 2 (solid line). The single-channel detection probabilities are depicted with dash-dotted and dotted lines. All the results refer to a moving target with $u_{r}=1 \times 10^{-3}, \mathrm{SCR}=10 \mathrm{~dB}, \mathrm{CNR}=10 \mathrm{~dB}$, and $\gamma_{c}=1$. The $P_{D}$ 's have been plotted versus the velocity values $u_{r, T}$ corresponding to the thresholds $\phi_{T}$.

In Figure 10, we have reported all the $P_{\mathrm{FA}}$ 's corresponding to the same operating conditions, adopting Strategy 1

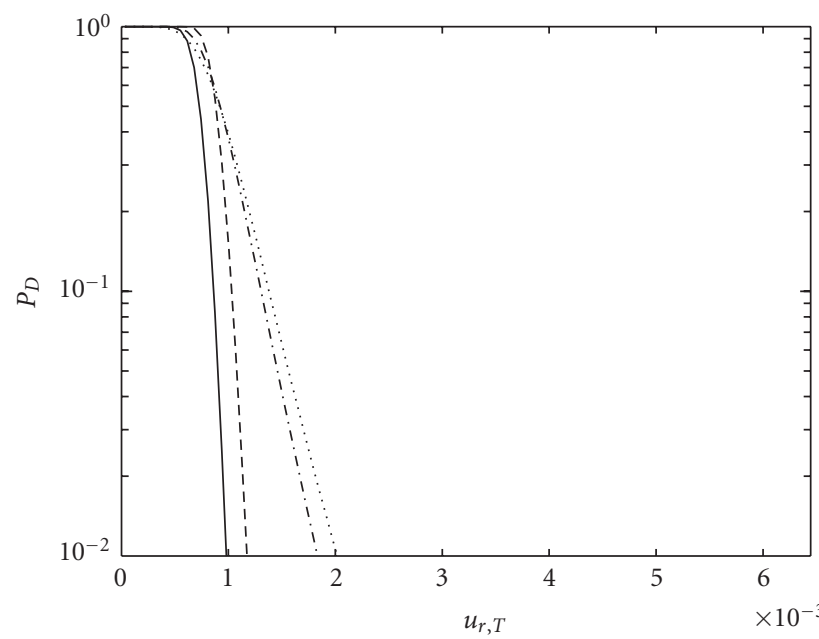

Figure 9: $P_{D}$ 's adopting Strategy 1 (dashed line) and Strategy 2 (solid line), in presence of a moving target with $u_{r}=0.001$, SCR $=\mathrm{CNR}=10 \mathrm{~dB}$, and $\gamma_{c}=1$. Single-channel $P_{D}$ 's are depicted with dash-dotted line (first baseline) and dotted line (second baseline).

(dashed line) and Strategy 2 (solid line). All the results are evaluated in absence of a moving target and for CNR $=10 \mathrm{~dB}$ and $\gamma_{c}=1$.

In Figure 11, Strategies 1 and 2 are compared in terms of $P_{\mathrm{FA}}$ and $P_{D}$. Following Strategy 2 , it can be found that a 


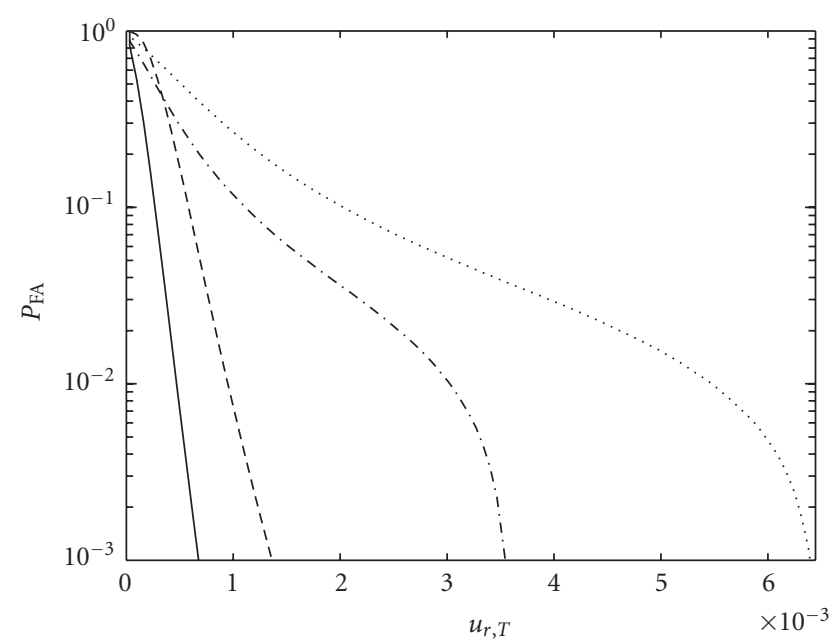

Figure 10: $P_{\mathrm{FA}}$ 's adopting Strategy 1 (dashed line) and Strategy 2 (solid line), in absence of moving targets, CNR $=10 \mathrm{~dB}, \gamma_{c}=$ 1. Single-channel $P_{\mathrm{FA}}$ 's are depicted with dash-dotted line (first baseline) and dotted line (second baseline).

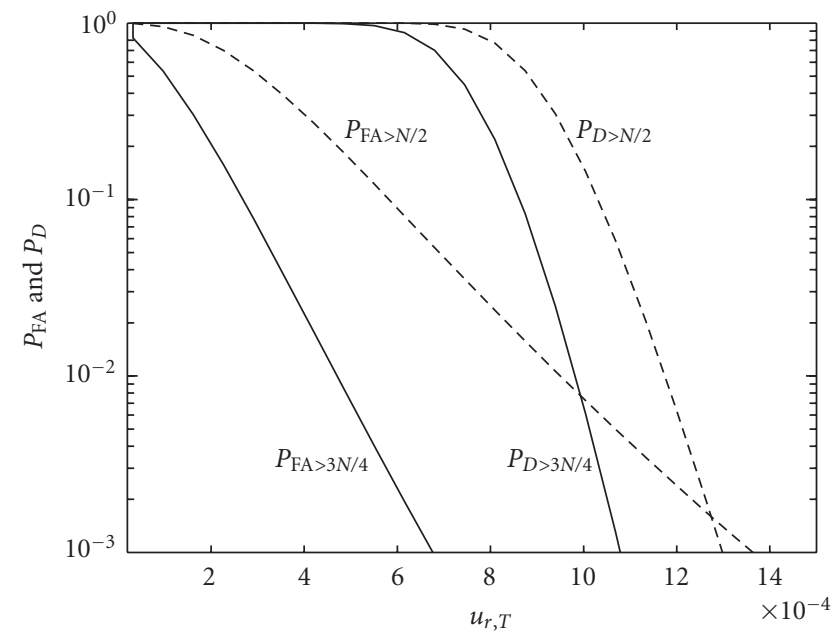

FIgUre 11: $P_{\mathrm{FA}}$ and $P_{D}$ adopting Strategy 1 (dashed line) and Strategy 2 (solid line). $u_{r}=1 \times 10^{-3}$, SCR $=\mathrm{CNR}=10 \mathrm{~dB}$, and $\gamma_{c}=1$.

threshold exists where performances are quite good with $P_{D}$ approaching 1 and $P_{\mathrm{FA}}$ approaching 0 .

Figure 11 has been plotted for a fixed velocity value $\left(u_{r}=1 \times 10^{-3}\right)$. When the velocity value changes, the detection probability changes, while the false alarm probability remains unchanged. In particular, considering Strategy 2, in Figures 12(a) and 12(b) it can be appreciated that, as expected, by increasing the velocity the detection probability increases.

To better show this effect, the detection probability versus velocity at fixed values of false alarm probability is shown in Figures 13(a) and 13(b) for CNR $=10, \gamma_{c}=1$, with SCR $=10$ (Figure 13(a)) and SCR $=20$ (Figure 13(b)) for two different fixed values of $P_{\mathrm{FA}}\left(10^{-2}\right.$ and $\left.10^{-3}\right)$.

It can be observed that in these examples a target can be detected with probability approaching 1 starting from normalized velocity values approximately equal to $u_{r}=10^{-3}$, also for $P_{\mathrm{FA}}=10^{-3}$. In this case, the phase thresholds corresponding to $u_{r}=10^{-3}$ and that guarantee $P_{\mathrm{FA}}=10^{-3}$ and $P_{D} \cong 1$ are $\phi_{T 1}=4 \pi b_{1} 10^{-3} / \lambda=0.49$ and $\phi_{T 2}=$ $4 \pi b_{2} 10^{-3} / \lambda=0.88$. In general, the phase thresholds depend on the minimum detectable velocity according to (3). The velocities values that can be detected reduce significantly when $P_{\mathrm{FA}}$ increases, or when the target RCS increases.

\section{MULTICHANNEL AT-InSAR MOVING TARGET RADIAL VELOCITY ESTIMATION}

As discussed in Section 2, the accuracy of the velocity estimation, obtainable with a given AT-InSAR system configuration, depends on the statistical model assumed for the target image, on the target radial velocity, and on the following parameters: SCR, CNR, $\gamma_{c}$, and $\gamma_{T 0}$.

We have already presented the multichannel system (multifrequency and/or multibaseline) configuration, providing the different phase measurements which are required to find a reliable solution for the detection and estimation problems $[13,15]$.

The ML estimation of the normalized radial velocity from multichannel data is given by

$$
\begin{aligned}
\hat{u}_{r} & =\underset{u_{r}}{\arg \max } L\left(u_{r}\right), \\
L\left(u_{r}\right) & =\prod_{\substack{n=1, \ldots, N \\
m=1, \ldots, M}}^{f_{\Phi}\left(\phi_{n, m} \mid u_{r} ; \lambda_{n}, b_{m}, \gamma_{c}, \gamma_{T 0}, \mathrm{SCR}, \mathrm{CNR}\right)},
\end{aligned}
$$

where $L(\cdot)$ is the multichannel likelihood function, obtained by multiplying the likelihood functions corresponding to the central frequency of the subbands and/or to the different baselines, and $\phi_{n, m}$ represent the wrapped phase values relative to the $N$ frequencies $c / \lambda_{n}$ and to the $M$ baselines $b_{m}$. The factorization (20) comes from the assumed statistical independence of the multichannel interferograms.

We evaluate numerically the Cramer-Rao lower bound (CRLB) of the estimated (normalized) velocity for the two different target statistical models considered above, and we estimate the target radial velocity using (20). We compare the CRLB with the root mean square error (RMSE) values. It has to be reminded that the CRLB represents the lower bound for the variance of the estimated parameter (the normalized radial velocity, in this case), whatever unbiased estimator working on the available set of data (the wrapped phases) may be considered [24].

We use the TerraSAR-X parameters introduced in Table 1 for the numerical simulation, and we consider a single-baseline system and a dual-baseline system $\left(b_{1}=\right.$ $1.2 \mathrm{~m}$ and $b_{2}=1.8 b_{1} \mathrm{~m}$ ), as in Section 4 . For each baseline we considered 2 subbands and 2 azimuth looks, in total 4 different channels [23]. For baseline $b_{1}$ the maximum radial velocity value that can be unambiguously detected is $\left|u_{r, \text { amb } 1}\right|=\lambda /\left(4 b_{1}\right)=6.5 \times 10^{-3}$ and for baseline $b_{2}$ is 


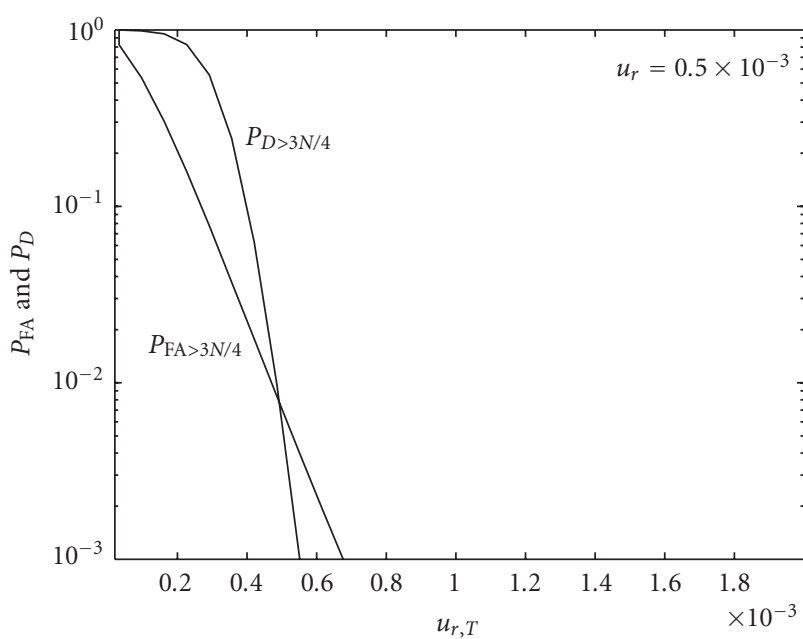

(a)

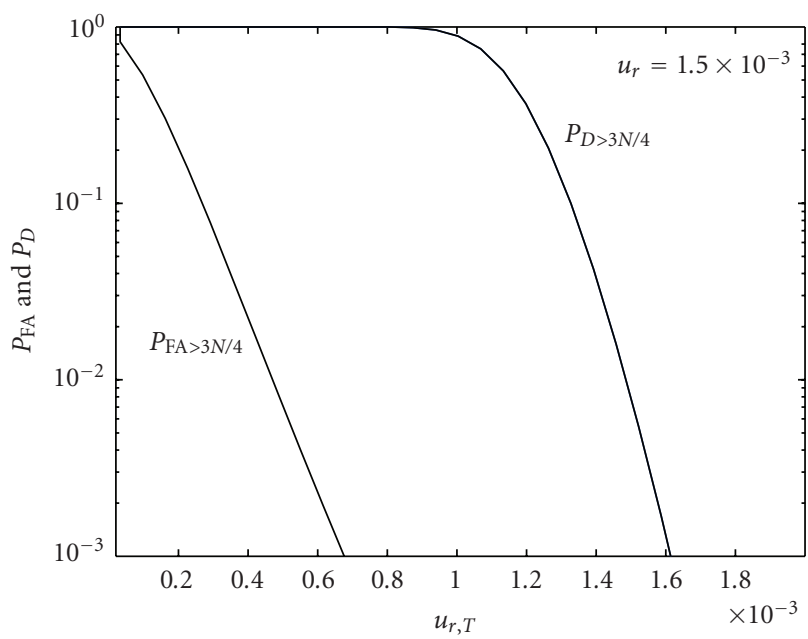

(b)

FIGURe 12: $P_{\mathrm{FA}}$ and $P_{D}$ adopting Strategy 2 for $\mathrm{SCR}=\mathrm{CNR}=10 \mathrm{~dB}, \gamma_{c}=1,0$; (a) $u_{r}=0.5 \times 10^{-3}$ and (b) $u_{r}=1.5 \times 10^{-3}$.

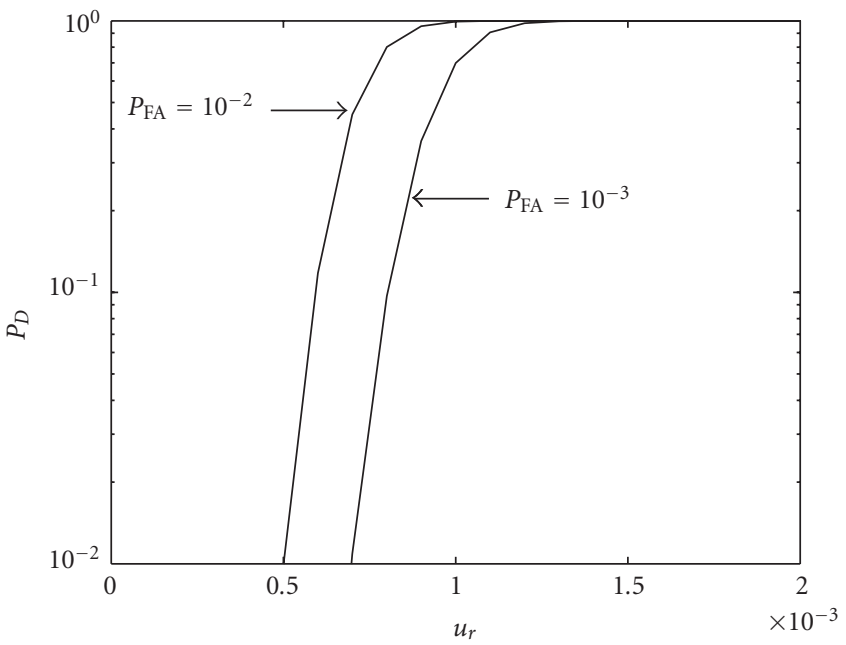

(a)

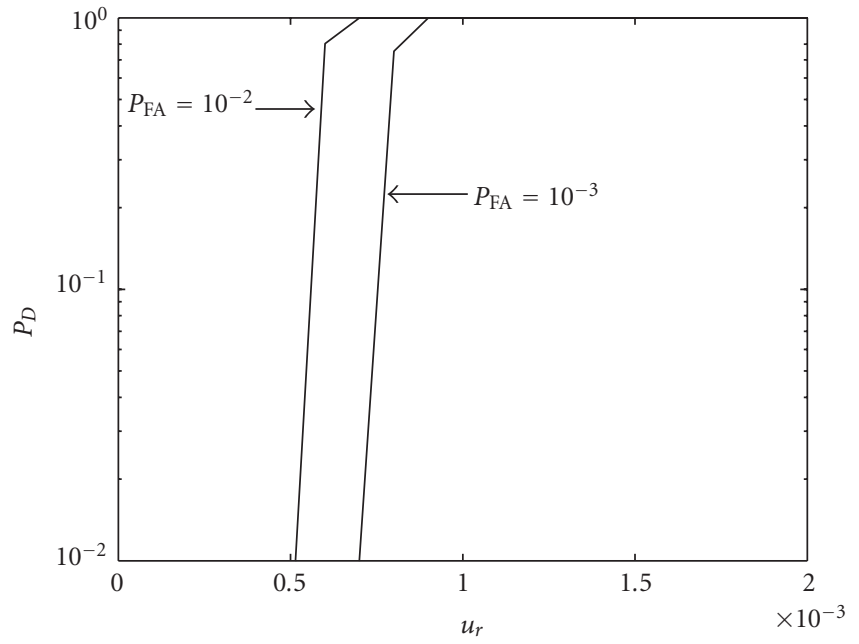

(b)

Figure 13: $P_{D}$ versus velocity for fixed values of $P_{\mathrm{FA}}\left(10^{-2}\right.$ and $\left.10^{-3}\right)$ for CNR $=10, \gamma_{c}=1$; (a) SCR $=10$ and $(\mathrm{b}) \mathrm{SCR}=20$.

$\left|u_{r, \text { amb2 }}\right|=\lambda /\left(4 b_{2}\right)=3.6 \times 10^{-3}$. A correspondence table between normalized and true radial velocity values in the TerraSAR-X case is reported in Table 2.

The CRLB ${ }^{1 / 2}$ and RMSE values (in logarithmic scale) for the deterministic case and for the Gaussian case, with $\gamma_{c}=1$, $\mathrm{SCR}=0,10$, and $20 \mathrm{~dB}, \mathrm{CNR}=10 \mathrm{~dB}$, and $b_{1}=1.2 \mathrm{~m}$, are reported in Figure 14. Of course, the SCR values are the ones obtained after the subband filtering (14).

The CRLB values vary with the normalized velocity $u_{r}$ to be estimated since the pdfs of the interferometric phase change with it (see Figures 3(c) and 4(c)), as shown in Section 2. In particular, in all cases considered, the CRLB values increase with the increasing of velocity. Estimation of velocity values is more accurate for small values of velocity (the CRLB are lower), and less accurate when velocity increases.
We observe also that, with the same SCR, CNR, and $\gamma_{c}$ values, the deterministic target model exhibits lower CRLB values than the Gaussian model. This effect is due to the different variance values corresponding to the deterministic and Gaussian cases for the same SCR, CNR, and $\gamma_{c}$ values (see Figures 3(c) and 4(c)). The larger the variance (the larger the phase noise), the larger the corresponding CRLB.

We observe further that in all considered cases the CRLB values decrease by increasing SCR under the same CNR and $\gamma_{c}$ values. Then, as expected, velocity estimation is more accurate when signal-to-clutter ratio is larger. It can be noted that the RMSE values for velocities far from the ambiguity value are only "slightly" larger than the CRLB ${ }^{1 / 2}$ ones and the RMSE values obtained using the deterministic model, likewise the CRLB ${ }^{1 / 2}$, are lower than the RMSE in the Gaussian case, as expected. This means that 4 channels 
TABLE 2: Correspondence between normalized and true velocity values in the TerraSAR-X case.

\begin{tabular}{lccccccccc}
\hline$u_{r}$ & $1 \times 10^{-4}$ & $4 \times 10^{-4}$ & $7 \times 10^{-4}$ & $1 \times 10^{-3}$ & $2 \times 10^{-3}$ & $3 \times 10^{-3}$ & $4 \times 10^{-3}$ & $5 \times 10^{-3}$ & $6 \times 10^{-3}$ \\
\hline$v_{r}[\mathrm{~m} / \mathrm{s}]$ & 0.76 & 3.04 & 5.32 & 7.60 & 15.2 & 22.8 & 30.4 & 38.0 & 45.6 \\
$v_{r}[\mathrm{~km} / \mathrm{h}]$ & 2.74 & 10.9 & 13.7 & 27.4 & 54.7 & 82.1 & 109.4 & 137.0 & 164.4 \\
\hline
\end{tabular}

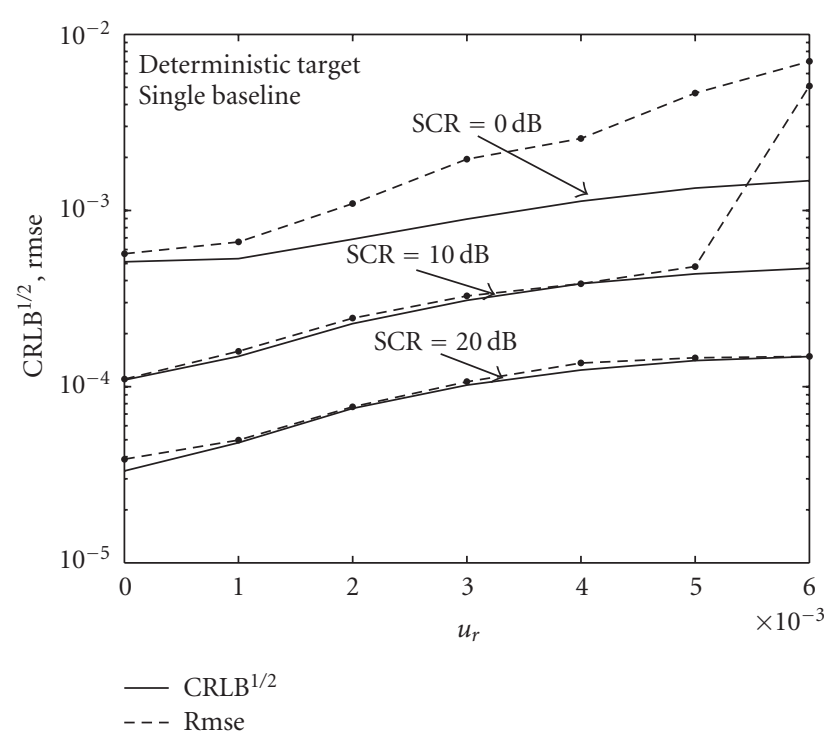

(a)

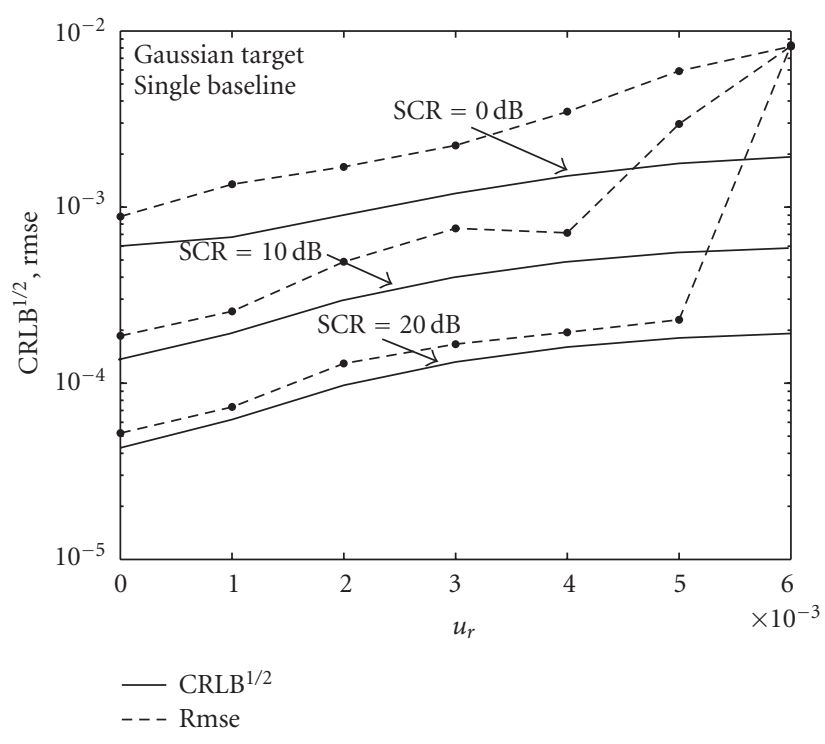

(b)

FIGURE 14: $\mathrm{CRLB}^{1 / 2}$ (solid line) and RMSE (dashed line) values (in log scale) of the estimated target radial normalized velocity, with CNR = $10 \mathrm{~dB}, \mathrm{SCR}=0,10,20 \mathrm{~dB}, \gamma_{c}=1$, for a single-baseline system $\left(b_{1}=1.2 \mathrm{~m}\right)$ for (a) a deterministic modeled moving target and (b) a Gaussian modeled moving target.

are sufficient, for the chosen values of CNR, SCR, and $\gamma_{c}$, to obtain quality performance very close to the best theoretical results when there are no ambiguity problems. Instead, it can be noted that the RMSE for velocities approaching the ambiguity value (from $4 \times 10^{-3}$ to $6 \times 10^{-3}$ ) and for low SCR tends to deviate from the CRLB values. This behavior is more pronounced in the Gaussian case.

In case we consider two baselines, we can obtain CRLB and RMSE values less variable with velocity and less sensitive to ambiguity problems. In particular, considering $b_{1}=1.2 \mathrm{~m}$ and $b_{2}=1.8 b_{1} \mathrm{~m}, \gamma_{c}=1, \mathrm{SCR}=0,10$, and $20 \mathrm{~dB}, \mathrm{CNR}=$ $10 \mathrm{~dB}$, we get the CRLB ${ }^{1 / 2}$ and RMSE curves (in logarithmic scale) shown in Figure 15, for the deterministic and Gaussian cases.

It can be appreciated that the CRLBs are reduced with respect to the single-baseline case, and that they are less variable with the velocity. Differently from the single-baseline system, in the dual-baseline system the RMSE values for all the velocities considered are very close to the CRLBs at least for high SCR values. Only for SCR $=0 \mathrm{~dB}$ it can be observed that a degradation of the estimation performances exists. The performance analysis shows that the considered ATInSAR system allows to estimate normalized radial velocity with RMSE of the order of $10^{-4}$ (using two baselines and 4 channels for baseline) even for SCR $=10 \mathrm{~dB}$. In traffic monitoring applications, when the moving targets are cars and trucks, the RCS can be of the order of, or larger than,
$100 \mathrm{~m}^{2}$. In this case, the SCR can be significantly larger than $10 \mathrm{~dB}$, allowing the application of this kind of sensors to practical situations.

\subsection{Multichannel ML velocity estimation algorithm robustness}

\subsubsection{Robustness with respect to the target model}

As discussed in Section 2, the deterministic target model is less tractable because, so far, no analytical statistical description has been obtained, but it is more realistic (targets of interest-cars, trucks-exhibit RCSs that do not vary significantly with the radar observation angles [19]). The Gaussian target model is less realistic (it is rare that cars and trucks response is a zero mean Gaussian signal which varies significantly inside the synthetic aperture), but it allows to perform the velocity estimation using analytical likelihood functions, since an analytical expression for the interferometric phase pdf is available with great advantages in terms of the computational efficiency. For this reason, we present the performance results in terms of RMSE values obtained using data generated with deterministic RCS, processed with the likelihood functions derived from the Gaussian model. In other words, we process the AT-InSAR data with a model different from the actual one used to simulate them. We compare again the single-baseline system 


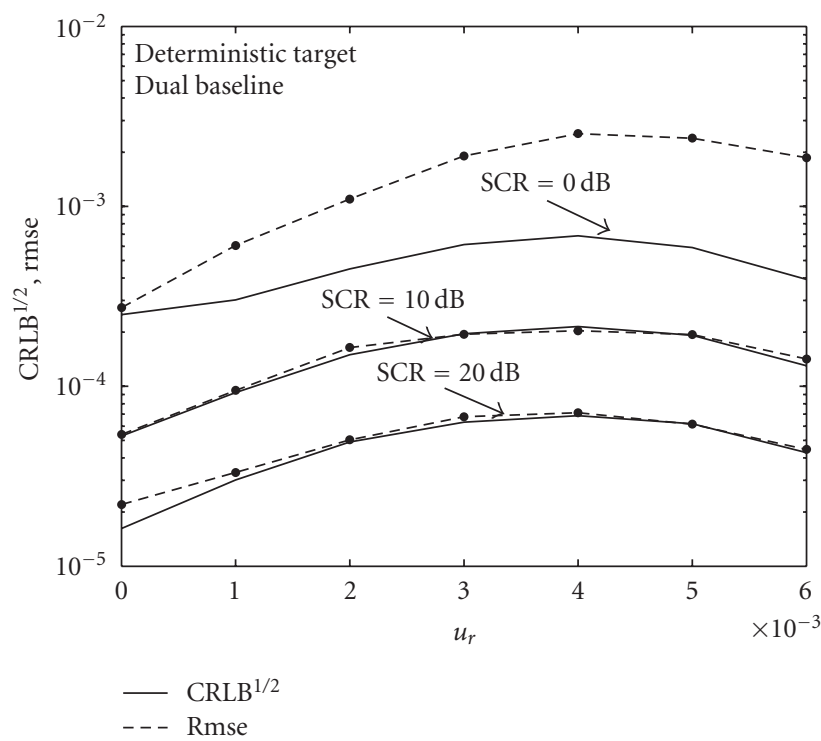

(a)

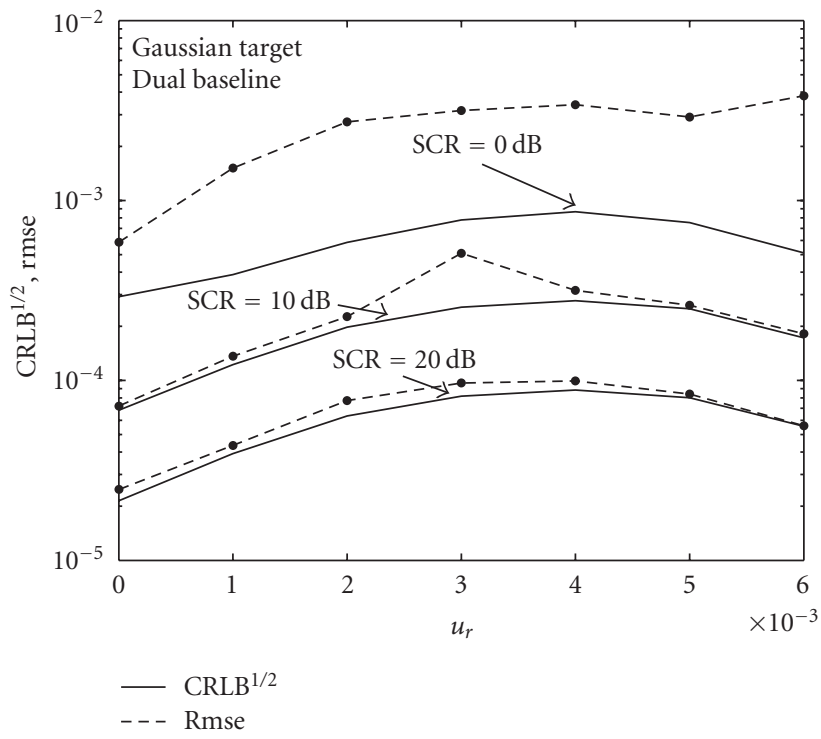

(b)

FIGURE 15: $\mathrm{CRLB}^{1 / 2}$ (solid line) and RMSE (dashed line) values (in log scale) of the estimated target radial normalized velocity, with CNR = $10 \mathrm{~dB}, \mathrm{SCR}=0,10,20 \mathrm{~dB}, \gamma_{c}=1$, for a dual-baseline system $\left(b_{1}=1.2 \mathrm{~m}, b_{2}=1.8 b_{1} \mathrm{~m}\right)$ for (a) a deterministic modeled moving target and (b) a Gaussian modeled moving target.

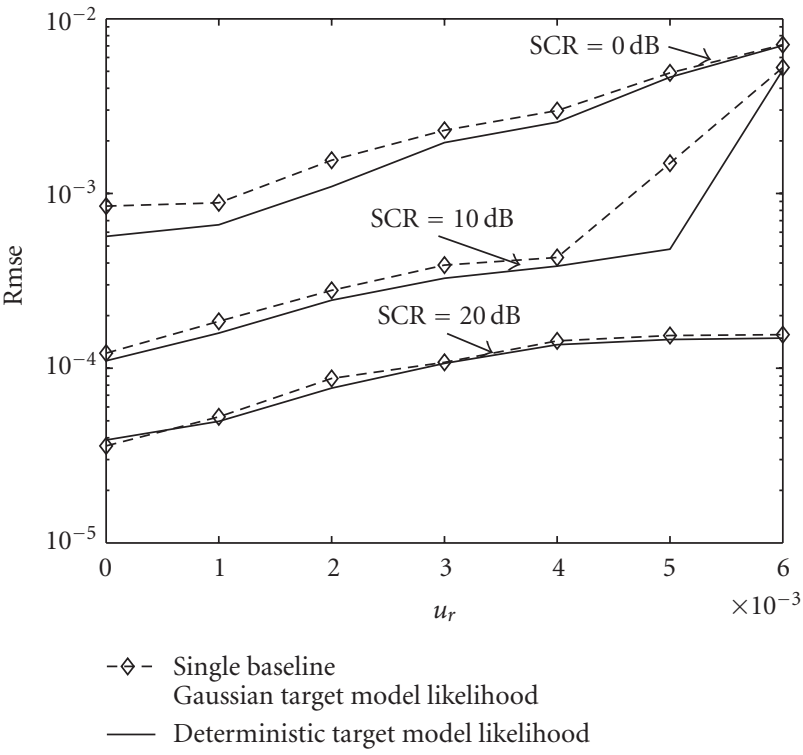

(a)

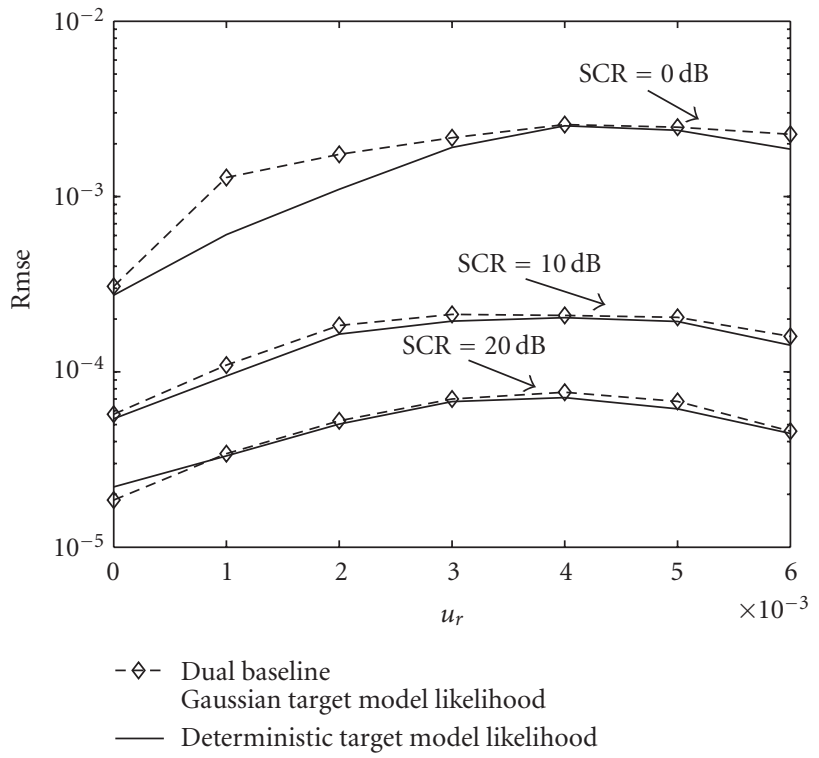

(b)

FIGURE 16: RMSE values of the estimated target radial normalized velocity, for a deterministic target likelihood (solid line) and RMSE values for a Gaussian target likelihood (dashed line), in log scale, with CNR $=10 \mathrm{~dB}, \mathrm{SCR}=0,10,20 \mathrm{~dB}, \gamma_{c}=1$ and for (a) a single-baseline system $\left(b_{1}=1.2 \mathrm{~m}\right)$ and (b) for a dual-baseline system $\left(b_{1}=1.2 \mathrm{~m}, b_{2}=1.8 b_{1} \mathrm{~m}\right)$.

with the dual-baseline one. The RMSE values for CNR = $10 \mathrm{~dB}, \mathrm{SCR}=0,10,20 \mathrm{~dB}$, and $\gamma_{c}=1$ for a deterministic modeled moving target obtained using a likelihood function derived from the deterministic and the Gaussian models are reported in Figure 16, for (a) the single-baseline system $\left(b_{1}=1.2 \mathrm{~m}\right)$ and (b) the dual-baseline system $\left(b_{1}=1.2 \mathrm{~m}\right.$, $\left.b_{2}=1.8 b_{1} \mathrm{~m}\right)$.
It can be noted, quite surprisingly, that the RMSE values processed with the likelihood functions derived from the Gaussian model are very close to the ones relative to the deterministic case. In such a way it is possible to process efficiently actual data with a tractable estimation algorithm without impairing significantly the estimation performance. 


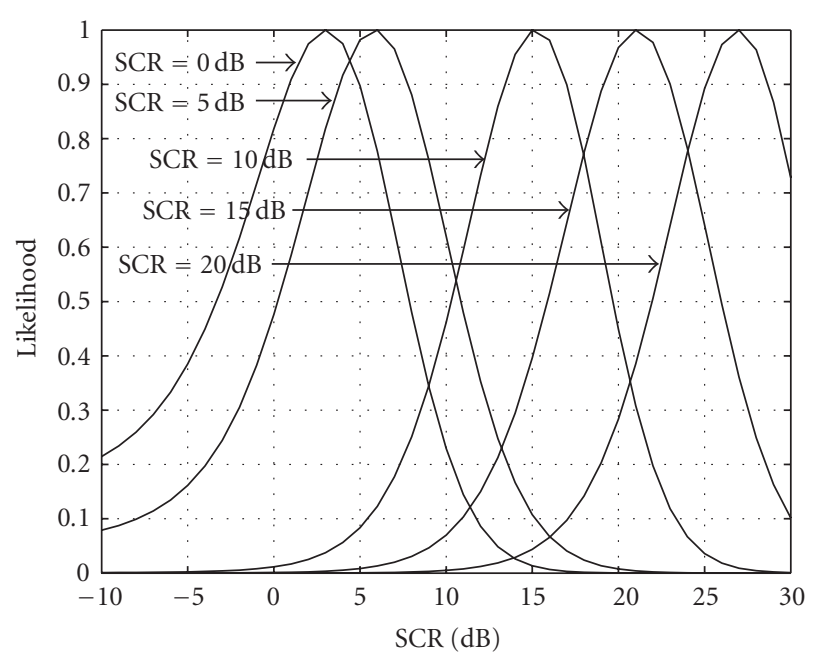

(a)

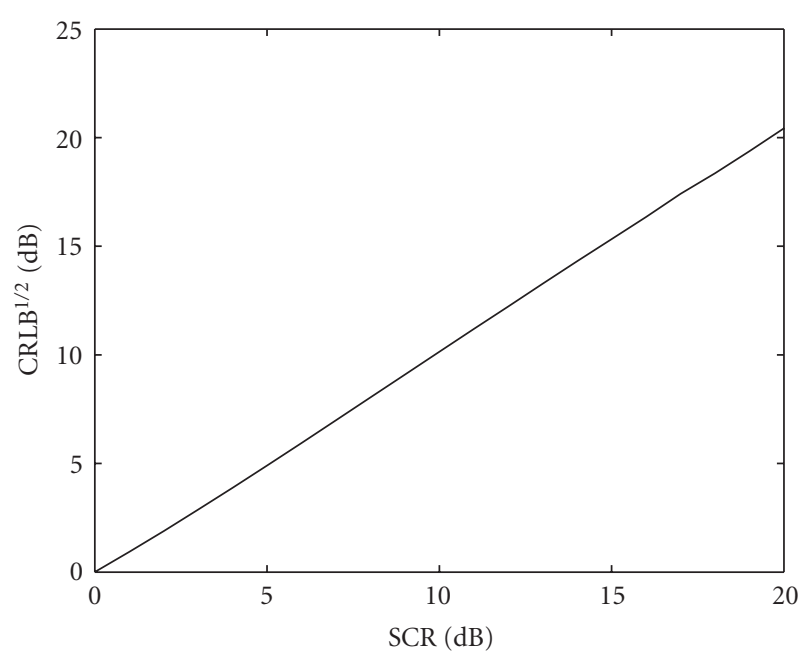

(b)

FIgURE 17: (a) Likelihood functions (21) versus SCR for a fixed radial velocity value; (b) square root of the CRLB for SCR.

TABLE 3: RMSE values of the estimated radial velocity relative to data generated with different values of SCR using the target deterministic RCS model processed with Gaussian RCS-derived likelihood functions, using, respectively, the nominal value of SCR (SCR $)$, the estimated (by (9)) value of SCR $\left(\mathrm{SCR}_{e}\right)$, and a fixed value of $\mathrm{SCR}=30 \mathrm{~dB}\left(\mathrm{SCR}_{f}\right)$. All other parameters $\left(\mathrm{CNR}=10 \mathrm{~dB}, \gamma_{c}=1\right.$, etc. $)$ are fixed.

\begin{tabular}{lcccccccccccc}
\hline & \multicolumn{3}{c}{$\mathrm{SCR}=5 \mathrm{~dB}$} & \multicolumn{3}{c}{$\mathrm{SCR}=10 \mathrm{~dB}$} & \multicolumn{3}{c}{$\mathrm{SCR}=15 \mathrm{~dB}$} & \multicolumn{3}{c}{$\mathrm{SCR}=20 \mathrm{~dB}$} \\
$u_{r}$ & $\mathrm{SCR}_{n}$ & $\mathrm{SCR}_{e}$ & $\mathrm{SCR}_{f}$ & $\mathrm{SCR}_{n}$ & $\mathrm{SCR}_{e}$ & $\mathrm{SCR}_{f}$ & $\mathrm{SCR}_{n}$ & $\mathrm{SCR}_{e}$ & $\mathrm{SCR}_{f}$ & $\mathrm{SCR}_{n}$ & $\mathrm{SCR}_{e}$ & $\mathrm{SCR}_{f}$ \\
\hline $1 e^{-3}$ & $3.77 e^{-4}$ & $4.24 e^{-4}$ & $4.14 e^{-4}$ & $1.73 e^{-4}$ & $1.82 e^{-4}$ & $2.26 e^{-4}$ & $9.45 e^{-5}$ & $9.68 e^{-5}$ & $1.23 e^{-4}$ & $5.07 e^{-5}$ & $5.23 e^{-5}$ & $6.33 e^{-5}$ \\
$2 e^{-3}$ & $5.37 e^{-4}$ & $5.55 e^{-4}$ & $6.48 e^{-4}$ & $2.97 e^{-4}$ & $2.99 e^{-4}$ & $3.54 e^{-4}$ & $1.46 e^{-4}$ & $1.51 e^{-4}$ & $1.93 e^{-4}$ & $8.62 e^{-5}$ & $8.98 e^{-5}$ & $1.08 e^{-4}$ \\
$3 e^{-3}$ & $1.30 e^{-3}$ & $1.15 e^{-3}$ & $1.21 e^{-3}$ & $3.58 e^{-4}$ & $3.73 e^{-4}$ & $4.75 e^{-4}$ & $1.96 e^{-4}$ & $1.97 e^{-4}$ & $2.71 e^{-4}$ & $1.05 e^{-4}$ & $1.08 e^{-4}$ & $1.42 e^{-4}$ \\
\hline
\end{tabular}

\subsubsection{Robustness with respect to the system parameters}

A further experiment directed to test robustness consists in varying the parameters of the model that are not perfectly known. In particular, while CNR and $\gamma_{c}$ can be easily obtained from the system characteristics (noise power of the SAR sensor) and from the processed SAR images (clutter power and coherence), the SCR value is unknown until the target is unknown (we do not a priori know its position or its RCS). Note also that it is not necessary to test robustness against CNR since, as highlighted in the comments to Figures 3 and 4, errors on it do not influence significantly the velocity estimation accuracy. Instead the role of SCR is quite determinant in the target velocity estimation procedure with respect to the other parameters, as shown by the pdf shapes and in the CRLB and RMSE behaviors. For this reason, we test the performance algorithm when the target RCS value is not a priori known.

First of all, we note that the SCR can be estimated from the data and the final estimation can be casted as a joint estimation of velocity and SCR:

$$
\begin{aligned}
{\left[\hat{u}_{r}, \mathrm{SCR}\right] } & =\underset{u_{r}, \mathrm{SCR}}{\arg \max } L\left(u_{r}, \mathrm{SCR}\right), \\
L\left(u_{r}, \mathrm{SCR}\right) & =\prod_{\substack{n=1, \ldots, N \\
m=1, \ldots, M}} \underbrace{f_{\Phi}\left(\phi_{n, m} \mid u_{r}, \mathrm{SCR} ; \vartheta\right)}_{\text {single channel likelihood function }},
\end{aligned}
$$

where $\vartheta$ denotes the following parameters $\lambda_{n}, b_{m}, \gamma_{c}=$ $1, \gamma_{T 0}=1, \mathrm{CNR}_{0}$ is computed from the data. We assume a Gaussian target model to derive the likelihood since it allows to perform the velocity estimation using analytical likelihood function and it does not degrade the estimation performance, as previously seen, even if the data are generated using a deterministic target model.

As far as the accuracy of the estimation of SCR is concerned, we show in Figure 17(a) some cuts (relative to different SCR values) of the likelihood function (21) versus SCR for a fixed radial velocity value. Note that the curves exhibit a similar shape since the axis of abscissas is in log scale, while in linear scale they would appear very different from each other, and their spreading increases by increasing SCR. Consequently, as the best theoretical obtainable accuracy (the Cramer-Rao lower bound (CRLB)) is directly connected to the spreading of the likelihood function, the accuracy worsens increasing the SCR. The complete behavior of the square root of the CRLB for SCR is presented in Figure 17(b) and confirms the mentioned trend.

We analyze also the system performance in terms of RMSE in the three following cases: (1) the estimation is performed by adopting for SCR the nominal value $\mathrm{SCR}_{n}$; (2) SCR is estimated in conjunction with the normalized radial velocity; (3) the estimation is performed by adopting for SCR a fixed value $\mathrm{SCR}_{f}$ different from the true one. In 
Table 3, we report the RMSE values of the estimated radial velocity using AT-InSAR images simulated with the system parameters reported in Table 1 , with $\mathrm{CNR}=10 \mathrm{~dB}, \mathrm{SCR}=$ $10 \mathrm{~dB}, \gamma_{c}=1$, and with normalized target velocities $u_{r}=$ $1 \times 10^{-3}, u_{r}=2 \times 10^{-3}, u_{r}=3 \times 10^{-3}$. The multichannel MLE algorithm (21) adopts a single baseline $\left(b_{1}=1.2 \mathrm{~m}\right)$ and 4 different channels (the 2 subbands and 2 azimuth looks used for the CRLB evaluation).

The best performance has been (of course) obtained using the nominal SCR values $\left(\mathrm{SCR}_{n}\right.$ column) but the adoption of the estimated SCR values through (21) $\left(\mathrm{SCR}_{e}\right.$ column) or the fixed one $\left(\mathrm{SCR}_{f}\right.$ column) do not impair significantly the velocity estimation performance, showing that there is a weak sensitivity of the velocity estimation from the SCR values. We have tested the robustness of the ML algorithm with respect to an unknown SCR in the singlebaseline system but we expect a similar behavior in the dualbaseline system.

\section{CONCLUSIONS}

In this paper, we presented the performance evaluation of multichannel AT-InSAR systems in terms of moving target detection ability and target radial velocity estimation accuracy. The analysis has been performed with different target statistical model and system parameters, such as radial velocity, SCR, CNR, and number of system channels. In particular, we compared a single-baseline system with a dualbaseline system. Regarding the detection process, the use of multichannel interferograms, after the application of a threshold stage to each channel, allows to adopt a binary integration to combine single-channel decisions. Such a strategy, compared with the one based on a single interferogram, provides better results in terms of simultaneous low values of $P_{\mathrm{FA}}$ and high values of $P_{D}$.

With reference to the target radial velocity estimation, two target models have been considered: deterministic target response and Gaussian target response. The first model is more realistic and applies to well-characterized targets, while the latter applies when the target RCS is not accurately known. The use of the Gaussian model in the velocity estimation procedure has proved to be particularly appropriate to the case of realistic datasets, since it allows to take into account the uncertainty on the knowledge of the target RCS. The mean square errors obtained using the Gaussian model are not very different from the lower bounds which are obtained using a deterministic model exploiting a very accurate knowledge of the target RCS. The estimation errors obtained using the Gaussian model exhibit a low sensitivity to the errors on the knowledge of the SCR value, which is a parameter difficult to be estimated in the absence of an accurate target characterization.

The analysis of performance which has been presented evidences that a single-baseline AT-InSAR system allows to obtain accurate radial velocity estimations approaching the CRLB also in the case of a small number of channels (only four, in the presented case) for velocities far from the ambiguity value, while to solve ambiguities at least a dualbaseline AT-InSAR system is required.
The present paper is focused on detection and estimation of interferometric phase data in order to highlight the performance improvement derived by the use of more than one channel (baseline). The use of more than two baselines would allow further performance improvement at the expenses of growing system complexity and cost.

\section{REFERENCES}

[1] H. Breit, M. Eineder, J. Holzner, H. Runge, and R. Bamler, "Traffic monitoring using SRTM along-track interferometry," in Proceedings of IEEE International Geoscience and Remote Sensing Symposium (IGARSS '03), vol. 2, pp. 1187-1189, Toulouse, France, July 2003.

[2] S. Hinz, F. Meyer, A. Laika, and R. Bamler, "Spaceborne traffic monitoring with dual channel synthetic aperture radar theory and experiments," in Proceedings of IEEE Computer Society Conference on Computer Vision and Pattern Recognition (CVPR '05), vol. 3, p. 7, San Diego, Calif, USA, June 2005.

[3] R. K. Raney, "Synthetic aperture imaging radar and moving targets," IEEE Transactions on Aerospace and Electronic Systems, vol. 7, no. 3, pp. 499-505, 1971.

[4] S. Barbarossa, "Detection and imaging of moving objects with synthetic aperture radar-part I: optimal detection and parameter estimation theory," IEE Proceedings of Radar and Signal Processing, vol. 139, no. 1, pp. 79-88, 1992.

[5] J. H. G. Ender, "Space-time processing for multichannel synthetic aperture radar," Electronics \& Communication Engineering Journal, vol. 11, no. 1, pp. 29-38, 1999.

[6] S. Barbarossa and A. Farina, "Space-time-frequency processing of synthetic aperture radar signals," IEEE Transactions on Aerospace and Electronic Systems, vol. 30, no. 2, pp. 341-358, 1994.

[7] J. H. G. Ender and D. Cerutti-Maori, "Position estimation of moving vehicles for space based multi-channel SAR/MTI systems," in Proceedings of the 6th European Conference on Synthetic Aperture Radar (EUSAR '06), pp. 1-4, Dresden, Germany, May 2006.

[8] S. Chiu, "Clutter effects on ground moving target velocity estimation with SAR along-track interferometry," in Proceedings of IEEE International Geoscience and Remote Sensing Symposium (IGARSS '03), vol. 2, pp. 1314-1319, Toulouse, France, July 2003.

[9] C. Elachi, Spaceborne Radar Remote Sensing: Applications and Techniques, IEEE Press, New York, NY, USA, 1988.

[10] R. M. Goldstein and H. A. Zebker, "Interferometric radar measurement of ocean surface currents," Nature, vol. 328, no. 6132, pp. 707-709, 1987.

[11] R. E. Carande, "Dual baseline and frequency along-track interferometry," in Proceedings of IEEE International Geoscience and Remote Sensing Symposium (IGARSS '92), pp. 1585-1588, Houston, Tex, USA, May 1992.

[12] C. W. Chen, "Performance assessment of along-track interferometry for detecting ground moving targets," in Proceedings of IEEE Radar Conference, pp. 99-104, Philadelphia, Pa, USA, April 2004.

[13] A. Budillon, V. Pascazio, and G. Schirinzi, "Multi-channel along track interferometry," in Proceedings of IEEE International Geoscience and Remote Sensing Symposium (IGARSS '04), vol. 4, pp. 2601-2603, Anchorage, Alaska, USA, September 2004.

[14] C. H. Gierull, "Statistical analysis of multilook SAR interferograms for CFAR detection of ground moving targets," IEEE 
Transactions on Geoscience and Remote Sensing, vol. 42, no. 4, pp. 691-701, 2004.

[15] A. Budillon, V. Pascazio, and G. Schirinzi, "Estimation of radial velocity of moving targets by along-track interferometric SAR systems," IEEE Geoscience and Remote Sensing Letters, vol. 5, no. 3, pp. 349-353, 2008.

[16] R. Bamler and P. Hartl, "Synthetic aperture radar interferometry," Inverse Problems, vol. 14, no. 4, pp. R1-R54, 1998.

[17] C. V. J. Jakowatz, D. E. Wahl, P. H. Eichel, D. C. Ghiglia, and P. A. Thompson, Spotlight-Mode Synthetic Aperture Radar: A Signal Processing Approach, Springer, Berlin, Germany, 1996.

[18] V. Pascazio, G. Schirinzi, and A. Farina, "Moving target detection by along-track interferometry," in Proceedings of IEEE International Geoscience and Remote Sensing Symposium (IGARSS '01), vol. 7, pp. 3024-3026, Sydney, Australia, July 2001.

[19] G. Palubinskas, H. Runge, and P. Reinartz, "Radar signatures of road vehicles," in Proceedings of IEEE International Geoscience and Remote Sensing Symposium (IGARSS '04), vol. 2, pp. 1498-1501, Anchorage, Alaska, USA, September 2004.

[20] J. Mittermayer and H. Runge, "Conceptual studies for exploiting the TerraSAR-X dual receive antenna," in Proceedings of IEEE International Geoscience and Remote Sensing Symposium (IGARSS '03), vol. 3, pp. 2140-2142, Toulouse, France, July 2003.

[21] R. V. Ostrovityanov and F. A. Basalov, Statistical Theory of extended Radar Targets, Artech House, Dedham, Mass, USA, 1985.

[22] J. S. Daba and M. R. Bell, "Statistics of the scattering cross-section of a small number of random scatterers," IEEE Transactions on Antennas and Propagation, vol. 43, no. 8, pp. 773-783, 1995.

[23] A. Budillon, G. Ferraiuolo, V. Pascazio, and G. Schirinzi, "Multichannel SAR interferometry via classical and Bayesian estimation techniques," Eurasip Journal on Applied Signal Processing, vol. 2005, no. 20, pp. 3180-3193, 2005.

[24] S. M. Kay, Fundamentals of Statistical Signal Processing: Estimation Theory, Prentice-Hall, Upper Saddle River, NJ, USA, 1993. 

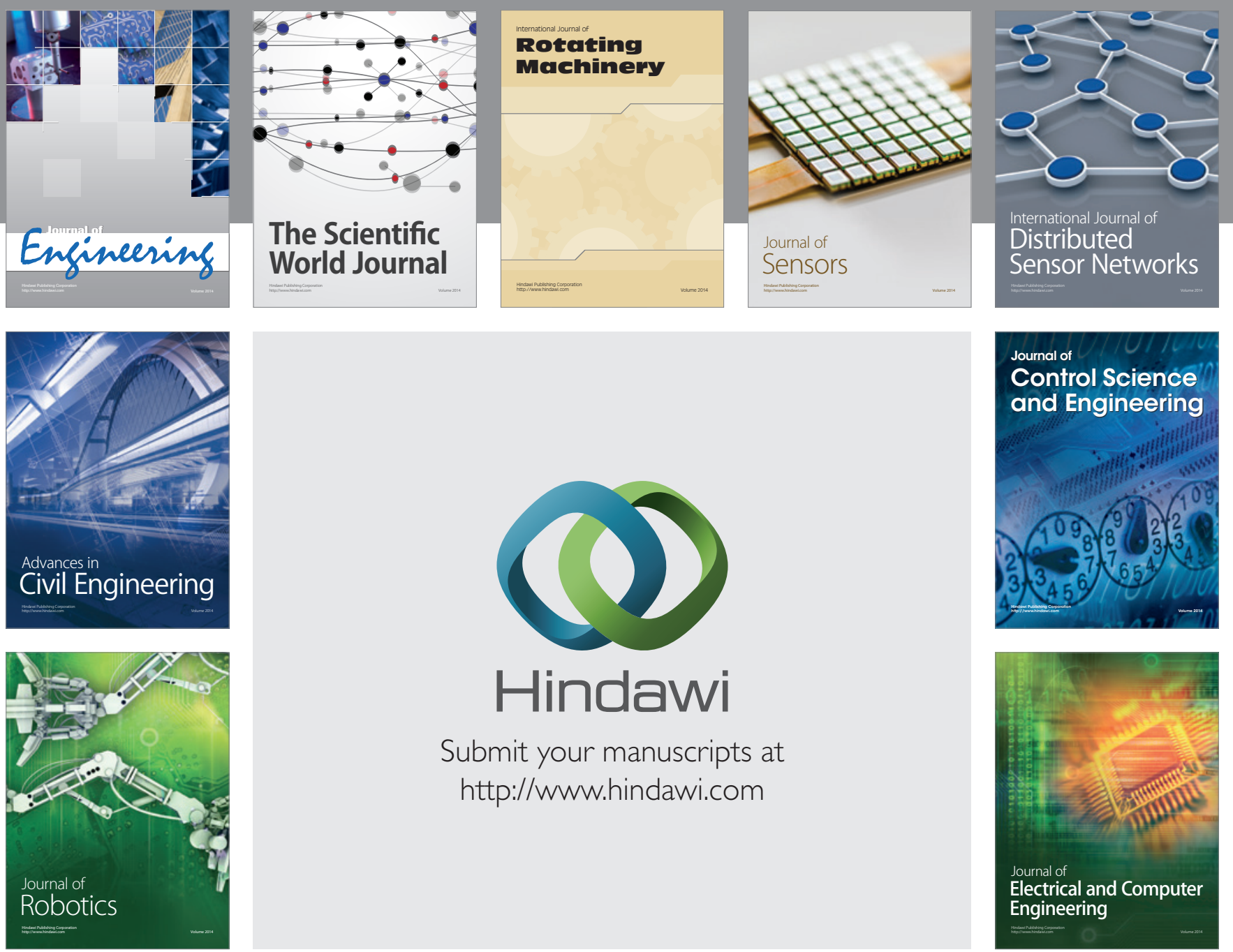

Submit your manuscripts at

http://www.hindawi.com
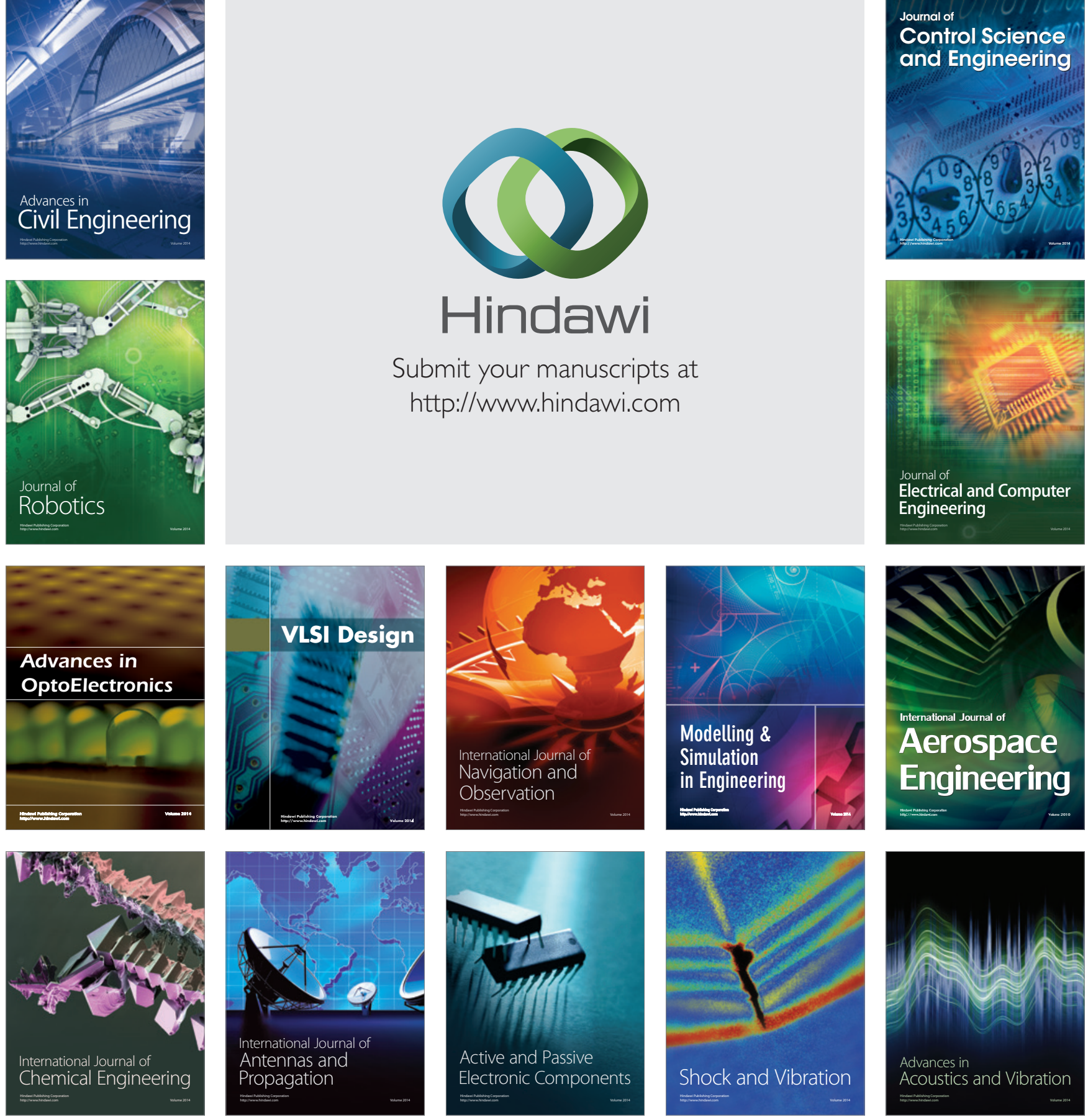